\title{
Vector extension of monogenic wavelets for geometric representation of color images
}

\author{
Raphaël Soulard, Philippe Carré and Christine Fernandez-Maloigne
}

\begin{abstract}
Monogenic wavelets offer a geometric representation of grayscale images through an AM/FM model allowing invariance of coefficients to translations and rotations. The underlying concept of local phase includes a fine contour analysis into a coherent unified framework. Starting from a link with structure tensors, we propose a non-trivial extension of the monogenic framework to vector-valued signals to carry out a non marginal color monogenic wavelet transform. We also give a practical study of this new wavelet transform in the contexts of sparse representations and invariant analysis, which helps to understand the physical interpretation of coefficients and validates the interest of our theoretical construction.
\end{abstract}

Index Terms-wavelet transform, color, analytic signal, monogenic signal, monogenic wavelets.

\section{INTRODUCTION}

Signal processing tools are accordingly suitable for audiovisual data thanks in part to their ability to model human perception. For several years, the large topic of defining visually relevant 2D tools gave rise to various geometric wavelet transforms designed to be local in space, direction and frequency [1]. In parallel, the 2D phase concept has gained much interest with new definitions for low-level vision and wavelet representations [2]-[5].

Research around phase concept began in the late 40's with the analytic signal [6], [7] giving the 1D 'instantaneous' phase by using a Hilbert transform. This tool is classical in 1D signal processing. In $2 \mathrm{D}$, the Fourier phase is the first known 2D phase concept, and it has been shown to carry important visual information in [8]. Afterwards, study of phase congruency [9], [10] proved that the phase can provide meaningful edge detection being invariant to intensity changes. A direct link between local phase and geometric shape of analyzed signal has been clearly established in [2], [11], [12]. In optics, image demodulation [13], [14] consists of building a 2D AM/FM model by extracting local amplitude and frequency (derivative of the phase) which in turn appears useful for texture segmentation.

The monogenic signal proposed by M. Felsberg [3] is the unifying framework that generalizes the analytic signal carrying out the 2D AM/FM model. As well as 2D Fourier atoms are plane waves defined by a $1 \mathrm{D}$ sinusoid and an orientation, the most natural 2D phase is basically a $1 \mathrm{D}$ phase with a local orientation. The Riesz transform is the key building block to define it - as the proper 2D generalization of the Hilbert transform [3], [15]. Note that the spiral quadrature phase transform of [14] used in optics is conceptually equivalent. Any image is viewed like local plane waves at different scales, with smoothly varying amplitude, phase, frequency and orientation. Because the phase concept is meaningful only for narrowband signals, it clearly has to go hand in hand with some multiscale decomposition such as a wavelet transform in order to analyze any class of signal. Among recent propositions of monogenic wavelets [5], [16], [17] we focus on this of [5] since it is tied to a minimally redundant perfect reconstruction filterbank. As we will see, monogenic wavelet coefficients have a direct physical meaning of local 2D geometry.

Despite this substantial research for the 2D case, signal tools are only applicable to grayscale images and hardly generalize to the

XLIM-SIC Department UMR CNRS 7252, University of Poitiers, France. Manuscript received January 7, 2012; revised May 30, 2012. vector-valued case. Yet analyzing color data is essential for quality control in different areas like agro-business, cosmetology or auto industry for example. Unfortunately, processing of color images is most often based on a marginal scheme that is applying scalar tools separately on each color channel [18]. Marginal schemes can produce unwanted false colors because they do not consider color entities. In the case of monogenic analysis, three independent orientations and phases would be extracted at every position and would have no physical meaning. The other circumvention is to use scalar tools on the intensity of a color image. Then the problem is a clear loss of information making impossible the detection of some contours for instance. Actually the color geometry information is spread over all color channels hence a real need for vector tools. A very recent color extension of Felsberg's work can be found in the literature [19]. We proposed its wavelet counterpart in a previous work [20] to carry out a non-marginal representation relying on a vector extension of CauchyRiemann equations that are the fundamental basis of the monogenic signal. This definition needs to improve its underlying phase concept. This paper goes further by proposing a new fully interpretable color monogenic analysis based on a link between the Riesz transform and differential geometry.

Differential approaches have a favorable algebraic framework to clearly define true vector tools through the vector structure tensor, popularized by Di Zenzo in 1986 [21]. These methods are based on estimation of image's gradient and rely on the assumption that resolution is 'sufficient'. Such methods yield remarkable geometric analysis and structure preserving regularization of color images [22], [23]. We propose to get the best of both worlds by considering the structure tensor based geometric analysis that is intrinsic to the monogenic framework.

We define in this paper a physical interpretation driven color extension of the grayscale monogenic wavelet transform by Unser $e t$ al. [5]. A few different approaches to wavelet analysis of multi-valued images may be retained in the literature. A vector lifting scheme is proposed in [24] for compression purpose, as well as wavelets within the triplet algebra in [25], but these separable schemes do not feature any geometric analysis, in contrast to our non separable approach allowing isotropy and rotation invariance. The multiwavelet framework yields generalized orthogonal filterbanks for multi-valued signals [26] but seems still limited to non-redundant critically sampled filterbanks. The connection with monogenic analysis is not yet apparent contrary to wavelet frames of [5]. None of these works make use of the phase concept that is central to this paper. In a previous work we have proposed a quaternionic filterbank [27], [28] for color images based on the quaternion color Fourier transform of [29]. We have observed that this quaternion formalism is impeding for proper physical interpretation of the data. The present contribution is a new step in this long line of works trying to propose a physical/signal framework for color images.

The paper will start with the recent and less recent definitions around the analytic/monogenic concepts in section II. Then section III will consist in proposing new definitions of color analytic/monogenic signal. Finally, the non-marginal color monogenic wavelet transform will be defined together with a practical study of the interpretation 
and use of wavelet coefficients in section IV.

Special Notations:

- Complex numbers: $z=\Re\{z\}+\boldsymbol{j} \Im\{z\}=|z| e^{\boldsymbol{j} \arg \{z\}}$.

- 2D coordinate in bold: $\boldsymbol{x}=\left[\begin{array}{ll}x_{1} & x_{2}\end{array}\right]^{\top}$ in the space domain, $\boldsymbol{\omega}=\left[\begin{array}{ll}\omega_{1} & \omega_{2}\end{array}\right]^{\top}$ in the fourier domain.

- Fourier transform: $s \stackrel{\mathcal{F}}{\longleftrightarrow} \hat{s}$ means that $\hat{s}$ is the Fourier transform of $s$.

- max: Maximum absolute value of a displayed dataset (used to normalize some illustrations).

\section{ANALYTIC AND MONOGENIC ANALYSES}

This section recalls existing definitions around the analytic signal and the monogenic signal. Provided analysis can be viewed like the demodulation of a 1D (resp. 2D) signal in amplitude, phase and frequency, based on the use of Hilbert (resp. Riesz) transform. Interpretation in terms of local geometry will be emphasized. The multiscale aspect will be presented through an overview of existing analytic wavelets followed by a more detailed description of the monogenic wavelets by Unser et al. [5]. Note that we present it from a rather practical point of view so we refer the reader to references provided in each subsection for further details.

\section{A. The analytic signal (1D)}

The analytic signal is defined in [6], [7] and can also be found in some general signal processing manuals as a classical tool. Given a scalar 1D signal $s(t)$, the associated complex-valued analytic signal $s_{A}(t)$ reads:

$$
s_{A}(t)=s(t)+\boldsymbol{j}\{\mathcal{H} s\}(t)=A(t) e^{\boldsymbol{j} \varphi(t)}
$$

where $\{\mathcal{H} s\}$ is the Hilbert transform of $s$ :

$$
\{\mathcal{H} s\}(t)=p . v \cdot \int \frac{s(t-\tau)}{\pi \tau} d \tau \stackrel{\mathcal{F}}{\longleftrightarrow}-\boldsymbol{j} \operatorname{sgn}(\omega) \hat{s}(\omega)
$$

Amplitude is $A(t)=\left|s_{A}(t)\right|$ and $\varphi(t)=\arg \left\{s_{A}(t)\right\}$ is the instantaneous (local) phase, as illustrated figure 1. Instantaneous frequency is given by $\nu(t)=\frac{d \varphi}{d t}$.

Under certain conditions $s$ can be viewed like a modulated oscillation, and then the underlying model tied to the analytic signal allows reconstructing $s$ with:

$$
s(t)=A(t) \cos [\varphi(t)]
$$

which highlights the notion of modulation.

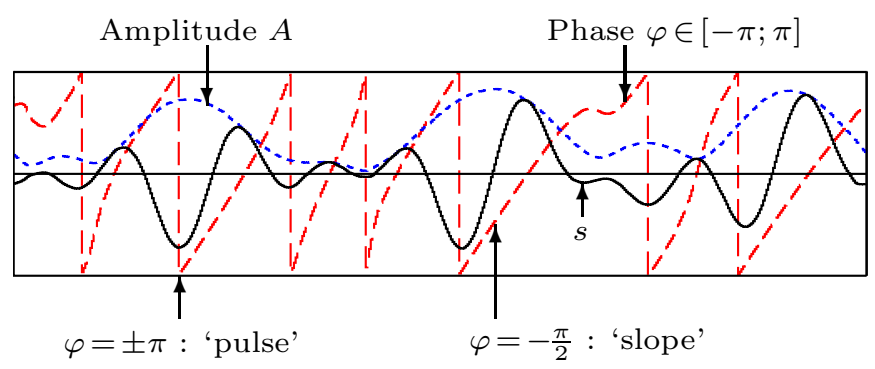

Fig. 1. Classical 1D analytic signal associated to a narrowband real (scalar) signal.

The growing interest on this tool within the image community is due to an alternative interpretation of amplitude, phase and frequency in terms of local geometric shape given in [2]. While amplitude conveys the relative presence of elements in the signal; phase describes the shape of those elements according to the correspondence given in table I. Hence the importance of defining a $2 \mathrm{D}$ analytic signal to extend this phase based analysis.
TABLE I

CORRESPONDENCE BETWEEN PHASE AND LOCAL SHAPE [2], [11].

\begin{tabular}{|l|c|c|c|c|}
\hline Phase $\varphi$ & 0 & $\frac{\pi}{2}$ & $\pm \pi$ & $-\frac{\pi}{2}$ \\
\hline Signal shape & $\bigwedge_{\longrightarrow}$ & $\bigwedge^{4}$ & $\wedge$ & \\
\hline
\end{tabular}

\section{B. The monogenic signal $(2 D)$}

The $2 \mathrm{D}$ extension of the analytic signal has been defined in several ways [2], [3], [30]. We are interested in the monogenic signal [3] because it is rotation invariant and its generalization is according to both fundamental definition and signal interpretation. Note that the underlying 2D phase concept was also studied in [11]. Given a 2D real (scalar) signal $s$, the associated monogenic signal $s_{M}$ is 3 -vector valued (instead of complex-valued in the $1 \mathrm{D}$ case) and must be taken in spherical coordinates:

$$
s_{M}=\left[\begin{array}{c}
s \\
\Re\{\mathcal{R} s\} \\
\Im\{\mathcal{R} s\}
\end{array}\right]=\left[\begin{array}{c}
A \cos \varphi \\
A \sin \varphi \cos \theta \\
A \sin \varphi \sin \theta
\end{array}\right]
$$

where $\mathcal{R} s$ is the complex-valued Riesz transform of $s$ :

$$
\{\mathcal{R} s\}(\boldsymbol{x})=p . v . \int \frac{\tau_{1}+\boldsymbol{j} \tau_{2}}{2 \pi\|\boldsymbol{\tau}\|^{3}} s(\boldsymbol{x}-\boldsymbol{\tau}) d \boldsymbol{\tau} \stackrel{\mathcal{F}}{\longleftrightarrow} \frac{\omega_{2}-\boldsymbol{j} \omega_{1}}{\|\boldsymbol{\omega}\|} \hat{s}(\boldsymbol{\omega})
$$

The monogenic signal is composed of the three following features:

$$
\begin{array}{ll}
\text { Amplitude: } & A=\sqrt{s^{2}+|\mathcal{R} s|^{2}} \\
\text { Orientation: } & \theta=\arg \{\mathcal{R} s\} \in[-\pi ; \pi[ \\
\text { 1D Phase: } & \varphi=\arg \{s+\boldsymbol{j}|\mathcal{R} s|\} \in[0 ; \pi]
\end{array}
$$

Note that we use a particular $\mathbb{C}$ embedding of the two components of the Riesz transform that is equivalent to the spiral phase quadrature transform defined by Larkin et al. in [14] and used by Unser et al. [5]. The first advantage of this formulation is the interpretation of the Riesz transform like a complex-valued filter. As the Hilbert transform can be viewed like an all-pass pure $\frac{\pi}{2}$-phase-shifting 1D filter, this embedding of the Riesz transform is an orientation dependent counterpart of it. The second advantage shown in [5] is that $\mathcal{R}$ is a unitary operator, which will help to define complex wavelet frames involving the Riesz transform.

We now discuss physical interpretation of analytic and monogenic analyses.

\section{Interpretation}

Both analytic and monogenic analyses give access to local amplitude and phase so as to model a signal by a modulated sinusoid $s=A \cos \varphi$. In the $2 \mathrm{D}$ case, $\varphi$ is understood along a local orientation $\theta$ analogous to some gradient direction data (more on this later).

The amplitude encodes local presence of geometrical elements while phase encodes the 1D local structure according to the correspondence in table I. Local phase is known to give a continuous model of the kind of discontinuity between the 'line' (resp. 'pulse' in 1D) and the 'edge' (resp. 'slope') in a unified framework [12]. Note that in [18], [31], amplitude is considered to carry some contrast information while local frequency (derivative of phase) conveys texture granularity. The signal model here is an $A$-strong structure that is oriented along $\theta$ and looking like rather an edge $(\varphi \approx \pm \pi / 2)$ or a line $(\varphi \approx 0$ or $\pi)$. Note that it excludes intrinsically $2 \mathrm{D}$ structures like junctions and corners. A recent extension of the monogenic framework - the conformal monogenic signal [32] - solves this problem but is out of the scope of this paper.

An important feature of this model is that the signal under analysis is expected to be narrowband. Equivalently, amplitude, phase and 
frequency must be smooth [13], [31]. A first reason comes from the meaning of phase. This data basically indicates a particular stage of some cyclic process - namely an oscillation. So for $\varphi$ to be interpretable the signal must be oscillating i.e. narrowband. Moreover, a narrowband signal is most likely to contain a unique frequency component; which ensures consistency of the analysis. Another reason is the mathematical constraint of Hilbert and Riesz transforms having a singularity at $\boldsymbol{\omega}=0$ in the Fourier domain which excludes all signals having a DC component. Note also that for the local frequency $\nu$ to exist, $\varphi$ and thus $s$ must be differentiable. In practice, the Riesz transform is used jointly with an isotropic bandpass filter [3].

This clearly suggests using monogenic analysis in a multiscale fashion through some subband decomposition in order to handle non narrowband signals as well.

\section{Scalar analytic wavelets: overview}

Gabor filters [13] and continuous scale-space [33] have been used in the literature to perform multiscale analytic/monogenic analysis. However these methods are overly redundant so they have to be compared with various differential approaches (PDE's, other scale spaces etc.) that already give efficient local geometric analysis. In contrast, filterbank and sampling approaches often involve small redundancy thanks in part to information/sampling theories. In this work we adopt this latter approach to multiscale monogenic analysis by focusing on wavelet transforms tied to perfect reconstruction filterbanks.

Here we recall some state of the art analytic wavelet transforms. These recent tools of representation allow some geometric multiscale analysis of grayscale images thanks to the phase concept brought by the analytic signal.

Note that these approaches are distinct from many definitions focusing on directionality such as Contourlets, 2D Complex Wavelets, Directionlets and adaptive lifting schemes. With analytic signal approaches, some redundancy is not set into a direction analysis; but rather into a phase data carrying richer geometric information and allowing some invariance.

The first proposition of analytic wavelets is for the 1D scalar case with the Dual-tree Complex Wavelet Transform (CWT) in 1999 [34]. It is a 1D discrete scheme consisting of two parallel filterbanks which filters are linked by Hilbert transforms. In fact, the Hilbert transforms are approximate because of discrete constraints. This method allows near shift-invariance of wavelet coefficients (shiftvariance is a famous problem of classical wavelets).

In 2004, a Quaternion Wavelet Transform (QWT) [4], [35] based on the quaternionic analytic signal of [2] is proposed for grayscale images. The quaternionic signal is a $2 \mathrm{D}$ generalization of the analytic signal that is prior to and maybe less convincing than the monogenic signal. Every quaternionic coefficient has a 3-angle phase that carries geometric information being complementary to the magnitude information from the modulus. The QWT of [4] consists of a separable implementation of the Dual-Tree algorithm, resulting in 4 parallel 2D filterbanks. A few applications of the QWT have been proposed [36][38] and take advantage of the shift invariance and the quaternionic phase to analyze images.

Finally in 2009 a Monogenic Wavelet Transform is proposed in [5]. This representation - specially defined for $2 \mathrm{D}$ signals - is a great theoretic improvement of the complex and quaternion wavelets; as well as the monogenic signal itself is an improvement of its complex and quaternion counterparts. To our knowledge this is the only monogenic wavelet transform that is tied to a minimally redundant filterbank in the present literature. In this paper this tool will be considered as the reference scalar (grayscale) 2D analytic wavelet transform. Let us present it in more details.

\section{E. The monogenic wavelet transform (MWT)}

The scheme of [5] performs multiresolution monogenic analysis by using two parallel filterbanks. One 'primary' transform tied to a real continuous wavelet frame and a so-called 'Riesz-Laplace' wavelet transform tied to a complex frame. Multiresolution analyses are built from the nearly isotropic polyharmonic B-spline of [39]:

$$
\beta_{\gamma} \stackrel{\mathcal{F}}{\longleftrightarrow}\left(\frac{4\left(\sin ^{2} \frac{\omega_{1}}{2}+\sin ^{2} \frac{\omega_{2}}{2}\right)-\frac{8}{3} \sin ^{2} \frac{\omega_{1}}{2} \sin ^{2} \frac{\omega_{2}}{2}}{\|\boldsymbol{\omega}\|^{2}}\right)^{\frac{\gamma}{2}}
$$

which is a valid scaling function. The wavelet for the 'primary' decomposition $\psi$ is a Mexican hat-like nearly isotropic function and the 'Riesz-Laplace' wavelet $\psi^{\mathcal{R}}$ is derived from it:

$$
\psi(\boldsymbol{x})=(-\Delta)^{\frac{\gamma}{2}} \beta_{2 \gamma}(2 \boldsymbol{x}) \quad \psi^{\mathcal{R}} \stackrel{\mathcal{F}}{\longleftrightarrow} \frac{\boldsymbol{j} \omega_{1}+\omega_{2}}{\|\boldsymbol{\omega}\|} \hat{\psi}(\boldsymbol{\omega})
$$

where the fractional Laplacian [40] is defined by ${ }^{1}$ :

$$
(-\Delta)^{\alpha} s \stackrel{\mathcal{F}}{\longleftrightarrow}\|\boldsymbol{\omega}\|^{2 \alpha} \hat{s}
$$

Let $\psi_{i, \boldsymbol{k}}(\boldsymbol{x})=2^{i} \psi\left(2^{i} \boldsymbol{x}-\boldsymbol{k} / 2\right)$ be the scaled and shifted version of $\psi$ (same for $\psi^{\mathcal{R}}$ ). It is shown in [5] that $\psi_{i, \boldsymbol{k}}$ and $\psi_{i, \boldsymbol{k}}^{\mathcal{R}}$ form two wavelet frames ensuring perfect reconstruction and orthogonality across scales. In addition, their operator-like behavior induces:

$$
\begin{aligned}
c_{i, \boldsymbol{k}}=\left\langle s, \psi_{i, \boldsymbol{k}}\right\rangle & =\left(\psi_{i} * s\right)\left(2^{-(i+1)} \boldsymbol{k}\right) \\
d_{i, \boldsymbol{k}}=\left\langle s, \psi_{i, \boldsymbol{k}}^{\mathcal{R}}\right\rangle & =\left\{\mathcal{R}\left(\psi_{i} * s\right)\right\}\left(2^{-(i+1)} \boldsymbol{k}\right)
\end{aligned}
$$

This means that wavelet coefficients form an exact monogenic signal at each scale. $c_{i, k}$ and $d_{i, k}$ are merged into 3-vectors and turned into polar coordinate according to eqs. (4) and (6) as illustrated figure 2. Interpretation of coefficients is the same as explained section II-C for

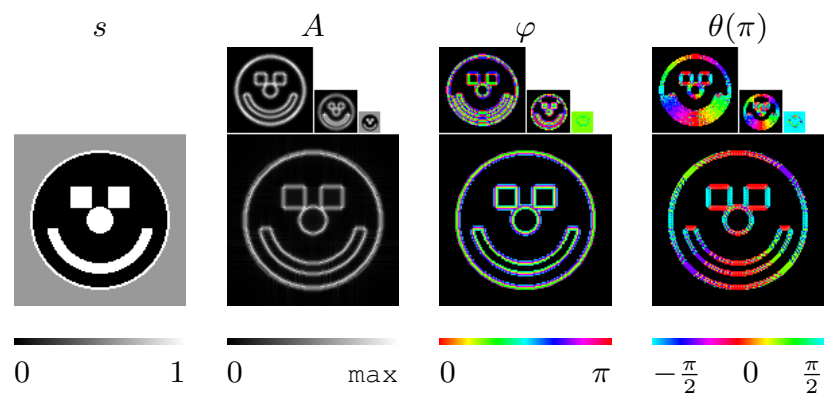

Fig. 2. Unser's MWT of image 'face'. Orientation $\theta$ is shown modulo $\pi$ for visual convenience. Phase values of small coefficients have no meaning so they are replaced by black pixels. We used $\gamma=3$ and the scales are $i \in\{-1,-2,-3\}$.

the monogenic signal but now concerns different scales of the image. Contrary to most used wavelet transforms this one is non-separable to nearly achieve rotation invariance of the monogenic framework. Both wavelet frames correspond to a dyadic pyramid filterbank needing only one 2D wavelet for each transform. Dyadic downsampling is done only at the low frequency branch leading to a total redundancy of 4:1. These particular pyramids designed in [41] feature a specific subband regression algorithm at the synthesis side.

In [5] a demonstration of AM/FM analysis is done with fine orientation estimation and gives very good results in terms of coherency and accuracy. The transform is also used in [42] for stereo vision.

\footnotetext{
${ }^{1}$ Note the particular case $\alpha=-1 / 2$ implying the isotropic low-pass filtering of frequency gain $1 /\|\boldsymbol{\omega}\|$; which will be used later.
} 
However, the monogenic wavelet representation clearly fails to handle color images. Applying it on intensity of an image would induce a serious loss of information around isoluminant contours. Using it marginally would have no more sense because independent geometric analyses would then be made on each color channel. Before going to our main proposition, let us quickly recall some background reference.

\section{F. Literature on color 'signal' tools}

Signal approaches to color images clearly suffer from a lack of tools. Some work must yet be mentioned.

Color algorithms can be processed by encoding the three color channels on the three imaginary parts of a quaternion as proposed by S. T. Sangwine and T. Ell in [29], [43] through their quaternion Fourier transform (this one is independent from the quaternion Fourier transform defined by T. Bülow for grayscale images [2]). We have studied it in a previous paper [28] for a precise definition of its properties in terms of color information. The representation is parameterized by a pure quaternion giving a direction of analysis in the color space. Depending on this choice, the interaction with the different real and imaginary parts of the spectral quaternionic domain is investigated. In most cases, the chosen parameter is on the gray line, so that the transform basically consists in two complex Fourier transforms used separately on the luminance part and the chrominance part of the image. We have also proposed a quaternion filterbank based on this concept [28], and came to the conclusion that the quaternion framework is impeding for an easy physical interpretation of the data.

A more recent work within the geometric algebra defines a non-marginal color extension of the monogenic analysis [19]. We extended it to a color monogenic wavelet transform in [20] and concluded that despite the non-marginality, the concept is not developed enough to obtain a clear physical interpretation. Furthermore, because this method applies the Riesz transform on the sum of color channels (due to prior mathematical justification), the geometric analysis fails to handle isoluminant contours.

In contrast, the present approach extends the monogenic wavelet transform so that a coherent color orientation is considered and the $1 \mathrm{D}$ phase interpretation is kept.

\section{COLOR MONOGENIC SIGNAL: A TENSOR APPROACH}

Given the great success of differential approaches in color vision [21], [22], we propose here to take advantage of the well established color structure tensor for a new color extension of monogenic analysis. The theoretical link between Riesz transform and gradient has already been studied and used in the grayscale case in [5], [44], [45]. We extend it to color thanks to the vector structure tensor concept. Then we discuss and define phase retrieval by oriented Hilbert analysis for color signals which completes the color phase concept. Physical interpretation of color monogenic features is given. Since the next section will extend the concept to a multiscale analysis, the present section is independent of any wavelet decomposition. We more generally assume that signals are bounded in frequency.

\section{A. The gradient-based structure tensor}

Here is recalled the classical gradient-based local analysis of grayscale images. A detailed explanation will be found in [46]. The gradient of an image $s$ is defined by:

$$
\nabla s=\left[\begin{array}{ll}
\frac{\partial s}{\partial x} & \frac{\partial s}{\partial y}
\end{array}\right]^{\top}=\left[\begin{array}{ll}
s_{x} & s_{y}
\end{array}\right]^{\top} \stackrel{\mathcal{F}}{\longleftrightarrow}\left[\begin{array}{lll}
\boldsymbol{j} \omega_{1} \hat{s} & \boldsymbol{j} \omega_{2} \hat{s}
\end{array}\right]^{\top}
$$

It points toward the direction of the local maximum variation of $s$; and its amplitude is relative to the strength of this variation. To get convinced of this, let us introduce the directional gradient:

$$
D_{\theta} s=\nabla s^{\top}[\cos (\theta) \sin (\theta)]
$$

This differential operator gives the '1D' local variation of $s$ in the direction $\theta$. We get an insight when rewriting it as follows:

$$
\begin{aligned}
& D_{\theta} s=\mathcal{N} \cos \left(\theta-\theta_{+}\right) \\
& \mathcal{N}=\sqrt{s_{x}^{2}+s_{y}^{2}} \quad(\text { gradient norm) } \\
& \theta_{+}=\arg \left\{s_{x}+\boldsymbol{j} s_{y}\right\} \quad \text { (gradient direction) }
\end{aligned}
$$

We see that the local variation is a cos-shaped function of the direction $\theta$ that reaches its maximum $D_{\theta_{+}} s=\mathcal{N}$ in the gradient direction. In other words, the gradient operator solves the maximization of the local variation with respect to direction ${ }^{2}$. Note that the minimum $D_{\theta_{+}+\pi}=-\mathcal{N}$ means an equally high variation in the opposite direction. These two opposite directions are merged into the same orientation according to the classical 'angle doubling' technique [11]:

$$
\theta_{+}=\frac{2 \arg \left\{s_{x}+\boldsymbol{j} s_{y}\right\}}{2}=\frac{\arg \left\{s_{x}^{2}-s_{y}^{2}+\boldsymbol{j} 2 s_{x} s_{y}\right\}}{2} \in\left[-\frac{\pi}{2} ; \frac{\pi}{2}\right]
$$

The edge strength $\mathcal{N}$ and orientation $\theta_{+}$form the well-known basic features for edge detection, as illustrated on the first row of Figure 3. However, the gradient analysis is only efficient for edge-like structures (see 'intrinsically 1D' or 'simple neighborhoods') - which is tied to the fact that it is done pointwise.

Let us now use the squared measure $s_{\theta}^{2}(\boldsymbol{x})=\left(D_{\theta} s(\boldsymbol{x})\right)^{2}$ in order to merge opposite directions, and consider its neighborhood defined by a window function $h(\boldsymbol{x})$, so as to carry out a more relevant oriented local variation [46]:

$$
\left(h * s_{\theta}^{2}\right)(\boldsymbol{x})=\int_{\mathbb{R}^{2}} h\left(\boldsymbol{x}-\boldsymbol{x}^{\prime}\right)\left\langle\nabla s\left(\boldsymbol{x}^{\prime}\right), \boldsymbol{u}\right\rangle^{2} d \boldsymbol{x}^{\prime}
$$

where $\boldsymbol{u}=\left[\begin{array}{ll}\cos (\theta) & \sin (\theta)\end{array}\right]$. This provides a quadratic form which maximization is known to be equivalent to find eigen- values/vectors of the underlying symmetric positive-definite matrix:

$$
T(s)=\left[\begin{array}{cc}
h * s_{x}^{2} & h * s_{x} s_{y} \\
h * s_{x} s_{y} & h * s_{y}^{2}
\end{array}\right]=\left[\begin{array}{cc}
T_{11} & T_{12} \\
T_{12} & T_{22}
\end{array}\right]
$$

called structure tensor. Eigenvalues can be derived analytically:

$$
\lambda_{ \pm}=\frac{1}{2}\left(T_{11}+T_{22} \pm \sqrt{\left(T_{22}-T_{11}\right)^{2}+4 T_{12}^{2}}\right)
$$

The eigenvector tied to $\lambda_{+}$is parallel to $\left[\cos \left(\theta_{+}\right) \sin \left(\theta_{+}\right)\right]$with $^{3}$ :

$$
\theta_{+}=\frac{1}{2} \arg \left\{T_{11}-T_{22}+j 2 T_{12}\right\}
$$

The second eigenvector is orthogonal to the first one. Note that we have directly the orientation $\theta_{+} \in\left[-\frac{\pi}{2} ; \frac{\pi}{2}\right]$ instead of direction. The strength of the maximum variation is given by:

$$
\left(h(\cdot) *\left(D_{\theta_{+}} s\right)^{2}(\cdot)\right)(\boldsymbol{x})=\lambda_{+}(\boldsymbol{x})
$$

while $\lambda_{-}$measures the minimum variation that is in the orthogonal orientation. This third feature enriches the local analysis and classically allows to discriminate isotropic structures, constant neighborhoods, junctions, corners... In the degenerate case where $h(\boldsymbol{x})=\delta(\boldsymbol{x})$, we get back to the gradient features with $\lambda_{+}=\mathcal{N}^{2}$,

${ }^{2}$ More precisely, it finds the best fit to the underlying linear model, with respect to the quadratic error [47]

${ }^{3}$ Depending on the paper one reads, both ' $T_{11}-T_{22}$ ' [21], [48]-[50] and ' $T_{22}-T_{11}$ ' [5], [46] can be found. According to us the former is the correct one (note that it is consistent with equation (17)). 


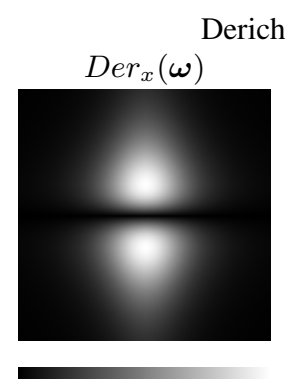

0

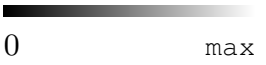

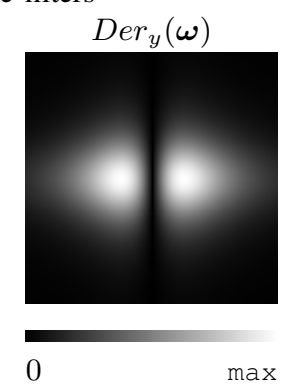
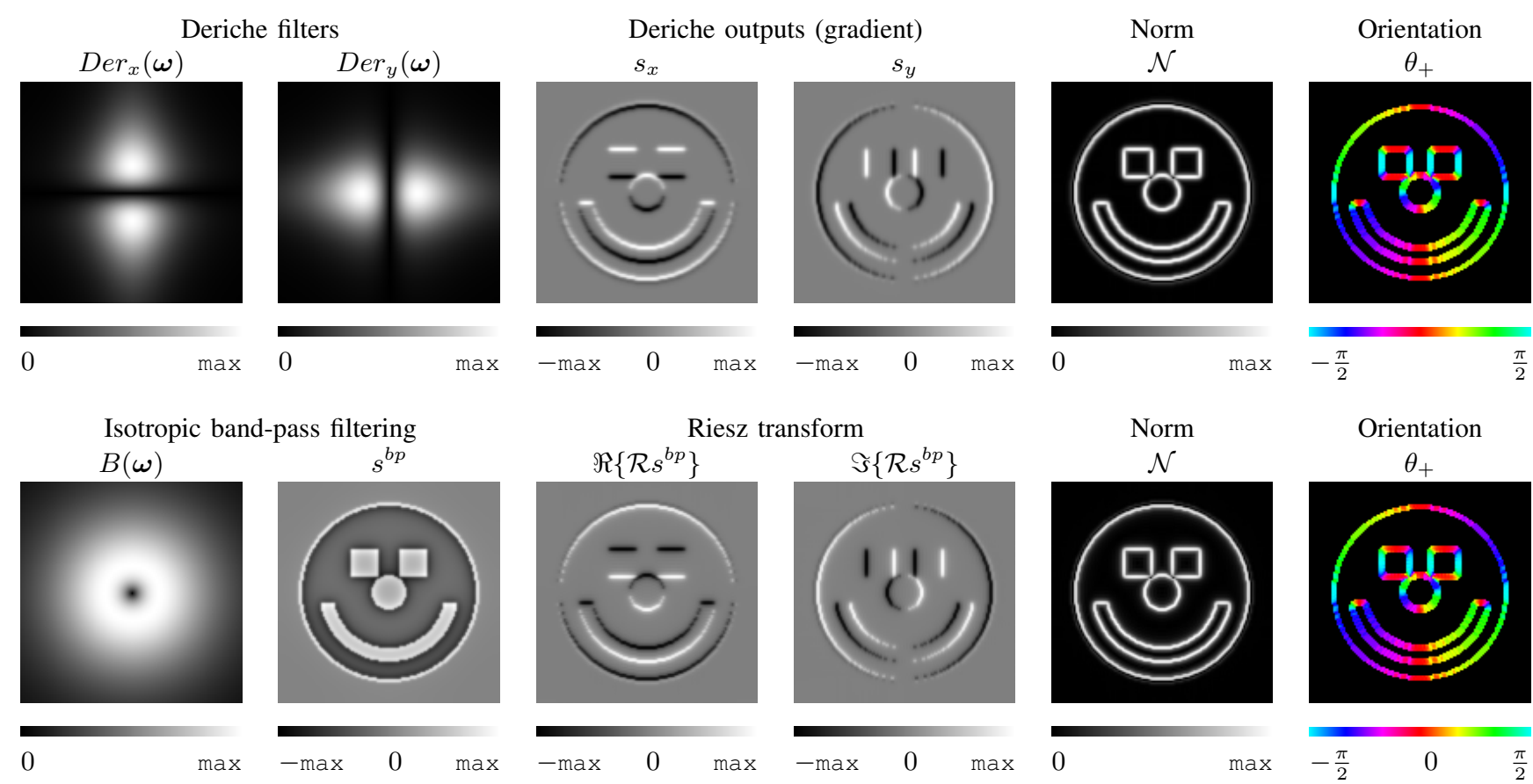

Fig. 3. Analogy between gradient analysis and Riesz analysis on test image $s$ already used in figure $2 . \mathcal{N}$ and $\theta_{+}$are according to eqs. (15) and (17).

$\lambda_{-}=0$, and orientation being identical to eq. (17) (due to the fact that in this case we have $T_{11} T_{22}=T_{12}^{2}$ ). In the general case, we still have this useful relation with gradient:

$$
\left(h(\cdot) * \mathcal{N}^{2}(\cdot)\right)(\boldsymbol{x})=\lambda_{+}(\boldsymbol{x})+\lambda_{-}(\boldsymbol{x})
$$

Now let us see that the Riesz transform can as well give rise to such analysis.

\section{B. Link between Riesz and gradient}

As already studied in [5], [45] the Riesz transform is very analogous to the gradient. More precisely, equations (5), (9) and (12) give:

$$
\mathcal{R} s=\left(-(-\Delta)^{-\frac{1}{2}} s_{x}\right)+\boldsymbol{j}\left(-(-\Delta)^{-\frac{1}{2}} s_{y}\right)
$$

Note that in spite of the Laplacian $\Delta$ being conceptually a highpass filter, $(-\Delta)^{-\frac{1}{2}}$ is low-pass - see eq. (9). As a result, $\mathcal{R}$ can be viewed either like the smoothed gradient of $s$ or like the gradient of a smoothed version of $s$. In [5], a Riesz counterpart of the structure tensor is derived to improve the Riesz analysis. The key is to see that $\mathcal{R} s$ maximizes the directional Hilbert transform response over the direction $\theta$ - in an identical fashion to the gradient. Based on this fact the whole structure tensor formalism can be derived with $\mathcal{R}$ replacing $\nabla$, so that we get the Riesz based tensor $T_{\mathrm{rz}}$ defined as follows:

$$
\begin{aligned}
& T_{\mathrm{rZ}}(s)=h *[\Re\{\mathcal{R} s\} \quad \Im\{\mathcal{R} s\}]^{\top}[\Re\{\mathcal{R} s\} \quad \Im\{\mathcal{R} s\}] \\
& =h *\left[\frac{\partial}{\partial x}\left((-\Delta)^{-\frac{1}{2}} s\right) \frac{\partial}{\partial y}\left((-\Delta)^{-\frac{1}{2}} s\right)\right]^{\top} \\
& {\left[\frac{\partial}{\partial x}\left((-\Delta)^{-\frac{1}{2}} s\right) \frac{\partial}{\partial y}\left((-\Delta)^{-\frac{1}{2}} s\right)\right]} \\
& =T\left((-\Delta)^{-\frac{1}{2}} s\right)
\end{aligned}
$$

that is nothing but the structure tensor of $(-\Delta)^{-\frac{1}{2}} s$ as defined in eq. (19). Finally, the Riesz features are equivalent to the structure tensor features of a smoothed version of $s$ :

$$
\begin{aligned}
|\mathcal{R} s| & \equiv \mathcal{N} \\
\arg \{\mathcal{R}\} & \equiv \theta_{+}
\end{aligned}
$$

Let us discuss the connection between $\mathcal{R}$ and $\nabla$ from a practical point of view.

Observations and Remarks: Most numerical implementations of gradient (Canny, Deriche, Shen-Castan, Sobel...) are basically directional band-pass filters resulting from the combination of differentiation (high-pass) and smoothing (low-pass). In the particular case of the Riesz transform, the frequency gain is 1 everywhere except at $\boldsymbol{\omega}=0$ - see eq. (5). The all-pass phase-shifting that is performed by the Riesz transform can be viewed like a generic variational analysis that is free from any scale selection. Then scale selection can be totally controlled by proper choice of band-pass filtering prior to the Riesz transform: this scheme is intrinsic to the monogenic analysis. Using some particular subband decomposition with a Riesz transform will therefore perform a multiscale gradient analysis which frequency selectivity will only depend on the chosen filterbank.

Figure 3 shows on the first row a classical gradient analysis with Deriche filters (Those filters optimize Canny's detector [51]). Second row is a Riesz analysis joint with an isotropic band-pass filtering. Deriche filters are parameterized with $\alpha=0.125 \times 2 \pi \sqrt{3}$, which correspond to a maximum Fourier response at $\omega=0.125 \times 2 \pi$. The band-pass filter is an isotropic difference of Poisson (DOP) wavelet with maximum response at the same frequency so that both analyses are at the same scale (Note that DOP filters have already been used in [52]). Filters are displayed with their normalized frequency gain. Outputs of filters and of Riesz transform are normalized around the gray level 128 to see positive (white) and negative (black) values. Riesz transform is computed with FFT which corresponds to a numerical approximation - this has a small impact since the impulse response decays like $1 / x^{2}$ (see eq. (5)). Orientation data is displayed in HSV color system with the hue encoding the angle. Value is set in $\{0,1\}$ with the thresholded norm so as not to display absurd angles, and Saturation is set to 1 everywhere.

We can see the perfect analogy between gradient and Riesz - which 
experimentally confirms the theoretic connection. It is clear that the difference of frequency response between $\mathcal{R}$ and $\nabla$ does not alter the local orientation analysis thanks to to the isotropy of $(-\Delta)^{-\frac{1}{2}}$. Finally, the building block of the monogenic analysis $\mathcal{R}$ performs the same efficient orientation analysis as a gradient. The advantage over the classical gradient is that it gives access to the local phase and frequency thanks to the monogenic concept that also includes the subband component $s^{b p}$ in a unified framework. It is now possible to extend it to color signals.

\section{Color Riesz, analysis}

The color structure tensor is the central tool of color differential approaches. The idea was first proposed by Di Zenzo in [21], and then further developed in [50]. This is now generally defined in Riemannian manifold embeddings [23] and used in general color imaging with PDE's and variational approaches [22]. Given a color image $s=\left(s^{\mathrm{R}}, s^{\mathrm{G}}, s^{\mathrm{B}}\right)$, consider its marginal gradients along $x$ and $y$ :

$$
\left[\nabla s^{\mathrm{R}}, \nabla s^{\mathrm{G}}, \nabla s^{\mathrm{B}}\right]=\left[s_{x}^{\mathrm{R}}, s_{y}^{\mathrm{R}}, s_{x}^{\mathrm{G}}, s_{y}^{\mathrm{G}}, s_{x}^{\mathrm{B}}, s_{y}^{\mathrm{B}}\right]
$$

The color structure tensor $M$ is defined as follows:

$$
M(s)=T\left(s^{\mathrm{R}}\right)+T\left(s^{\mathrm{G}}\right)+T\left(s^{\mathrm{B}}\right)=\left[\begin{array}{ll}
M_{11} & M_{12} \\
M_{12} & M_{22}
\end{array}\right]
$$

with

$$
\begin{aligned}
& M_{11}=h *\left(\left(s_{x}^{\mathrm{R}}\right)^{2}+\left(s_{x}^{\mathrm{G}}\right)^{2}+\left(s_{x}^{\mathrm{B}}\right)^{2}\right) \\
& M_{12}=h *\left(s_{x}^{\mathrm{R}} s_{y}^{\mathrm{R}}+s_{x}^{\mathrm{G}} s_{y}^{\mathrm{G}}+s_{x}^{\mathrm{B}} s_{y}^{\mathrm{B}}\right) \\
& M_{22}=h *\left(\left(s_{y}^{\mathrm{R}}\right)^{2}+\left(s_{y}^{\mathrm{G}}\right)^{2}+\left(s_{y}^{\mathrm{B}}\right)^{2}\right)
\end{aligned}
$$

Norm $\mathcal{N}$ and direction $\theta_{+}$of the maximum local variation are again obtained from eigenvalues and eigenvectors according to eqs. (20) and (21). The underlying measure of variation analogous to eq. (18) now involves Euclidean distances between neighboring vector-valued pixels.

We saw above that Riesz features are equivalent to gradient norm and direction so we straightforwardly obtain the following color Riesz features:

$$
\begin{array}{r}
\mathcal{N}=\sqrt{\left|\mathcal{R} s^{\mathrm{R}}\right|^{2}+\left|\mathcal{R} s^{\mathrm{G}}\right|^{2}+\left|\mathcal{R} s^{\mathrm{B}}\right|^{2}} \\
\theta_{+}=\frac{1}{2} \arg \left\{\sum_{\mathrm{c} \in\{\mathrm{R}, \mathrm{G}, \mathrm{B}\}} \Re\left\{\mathcal{R} s^{\mathrm{C}}\right\}^{2}-\Im\left\{\mathcal{R} s^{\mathrm{C}}\right\}^{2}\right. \\
\left.\quad+\boldsymbol{j} \sum_{\mathrm{C} \in\{\mathrm{R}, \mathrm{G}, \mathrm{B}\}} 2 \Re\left\{\mathcal{R} s^{\mathrm{C}}\right\} \Im\left\{\mathcal{R} s^{\mathrm{C}}\right\}\right\}
\end{array}
$$

Note that according to the monogenic framework [3] and to the color gradient by Di Zenzo [21], we restrict ourselves to gradient features; which are a degenerate case of the structure tensor where $h(\boldsymbol{x})=$ $\delta(\boldsymbol{x})$. The advantage of defining color Riesz features with differential geometry is the proper analysis of color discontinuities; as illustrated figure 4 . Note that only the RGB color space is considered, although it is well known to be a 'bad' space with respect to the human visual system, as well as highly correlated. However this is mathematically handy and the definition of color structure tensor on non-linear spaces like $L a b$ is not as well established, and is linked to the open topic of perceptual color distances. As we will see, choosing the RGB space will still allow performing satisfactory color image processing and analysis.

The figure 4 shows a band-pass filtering (same filter as figure 3) of a color image followed by the color Riesz analysis. Output of filtering $s$ and its Riesz transform components are 'subbands' i.e. oscillating at those locations where the original image has a discontinuity. As illustrated figure 5 (A), a color discontinuity in the original image $I(x)$ draws a certain direction $\vec{u}$ in the RGB cube. The resulting subband $s(x)$ locally oscillates along the color axis $\vec{u}(\mathbf{B})$. This data has negative values so it must be normalized to observe it as a color bitmap (C). Note that mid-gray $(128,128,128)$ actually encodes value $(0,0,0)$, which explains the gray background of subbands of figure 4 corresponding to uniform areas. Colors of illustrations of subbands in figure 4 are rather 'encoded 3-vectors' than actual colors.

Note the use of marginal band-pass filtering and marginal Riesz transform; which is the direct consequence of using a tensor approach i.e. based on a marginal gradient. However, final features $\mathcal{N}$ and $\theta_{+}$ are extracted in a non marginal way.

Figure 4 shows that the color Riesz analysis gives the proper orientation of all color contours, including isoluminant ones like the border between red and green disks that would disappear in an intensity-based scheme like this of our previous work [20] based on [19]. Note that we have two distinct notions of direction:

- Spatial orientation corresponding to the geometry of image content that is given by $\theta_{+}$from the Riesz analysis,

- Color direction in the RGB space, which is a feature of discontinuities independent from the Riesz analysis. This will be studied below.

Now that color Riesz features $\mathcal{N}$ and $\theta_{+}$are well defined, we can generalize the whole monogenic analysis to color. The last step is to build the 1D phase for color images.

\section{Color phase}

In the grayscale case, the analytic signal consists in combining a signal $s$ with a phase-shifted version of itself $(\mathcal{H} s$ or $|\mathcal{R} s|)$ to extract local amplitude $A$ and phase $\varphi$. In 2D, orientation is locally taken into account in the definition of the phase-shift so that $|\mathcal{R} s|$ is analogous to $\mathcal{H} s$ in the direction of maximum variation. With eq. (28) it turns out that this phase-shifted signal is in fact the Riesz gradient norm $\mathcal{N}$ which also holds in the color case with eq. (35). Our mathematical problem is that in color, $s$ is a 3-vector while $\mathcal{N}$ is still scalar. Fortunately, the Euclidean norm $\|s\|=\sqrt{\left(s^{\mathrm{R}}\right)^{2}+\left(s^{\mathrm{G}}\right)^{2}+\left(s^{\mathrm{B}}\right)^{2}}$ carries all needed information to compute meaningful amplitude and phase.

To get convinced, let us replace $s$ by its absolute value $|s|$ in the grayscale monogenic model (recall that $s$ is considered oscillating so $s \neq|s|)$ :

$$
\begin{aligned}
s & =\underbrace{\sqrt{s^{2}+\mathcal{N}^{2}}}_{A} \cos (\underbrace{\arg \{s+j \mathcal{N}\}}_{\varphi \in[0 ; \pi[}) \\
& =\underbrace{\sqrt{|s|^{2}+\mathcal{N}^{2}}}_{A} \cos (\underbrace{\arg \{|s|+j \mathcal{N}\}}_{\varphi_{2} \in\left[0 ; \frac{\pi}{2}[\right.}) \underbrace{s /|s|}_{\text {sign" }}
\end{aligned}
$$

We must separate the sign of $s$ as a new data, but $A$ is unchanged, and the new phase $\varphi_{2}$ is a restricted version of $\varphi$ to interval $\left[0 ; \frac{\pi}{2}\right]$. This restriction just reduces to an angle-wrapping problem. In the original model, a black line on white background has a $\pi$ phase while a white line on a black background has a 0 phase. Now both lines have a 0 phase but the former is along the 'black-white' axis while the latter is along the opposite 'white-black' axis. This axis information is encoded in the sign data. The same remark holds with edges from black to white and from white to black with $\pi / 2$ and $-\pi / 2$. We still keep the desired edge/line discrimination - as illustrated in table II - and still can differentiate the phase to get the local frequency. This means that the sign data is not needed to retrieve satisfactory physical interpretation of phase. The sign data does not encode any shape information but rather a direction in the space of gray levels (not to be confused with spatial direction given by $\theta$ ). 

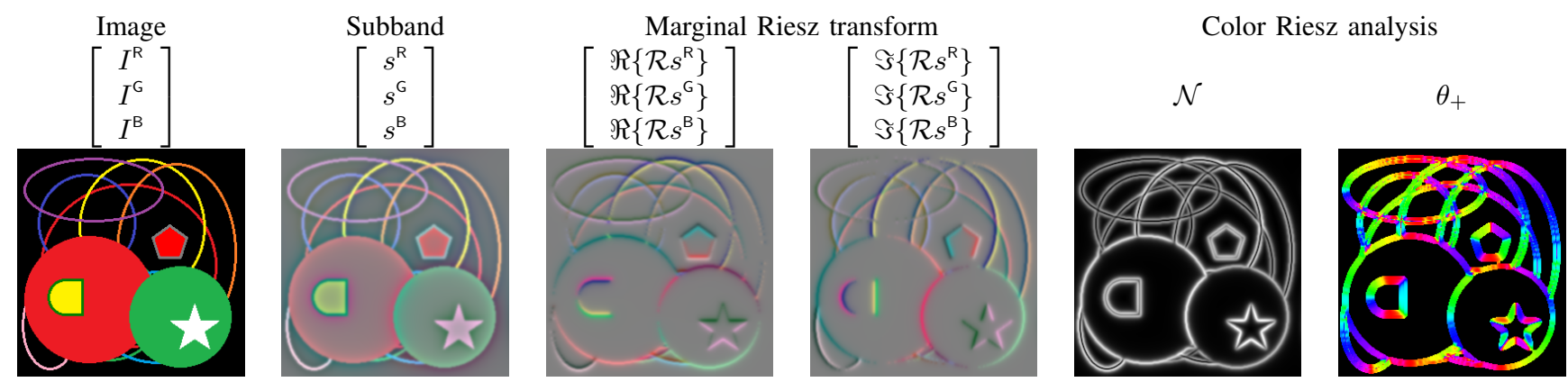

Fig. 4. Color Riesz analysis. From left to right: Color image, marginal band-pass filtering output, marginal Riesz transform ( 2 components), color Riesz features (norm and direction).

A: Color discontinuity (1D) in the RGB space $[0 ; 255]^{3}$ :

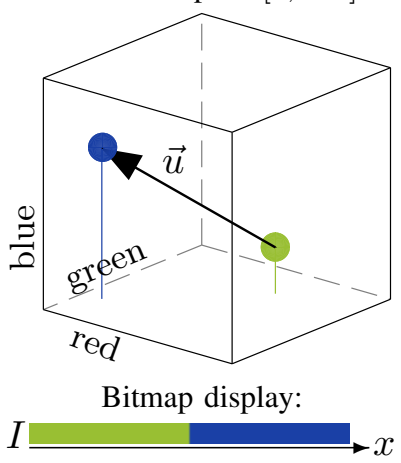

$x$
B: Band-pass filtering output in $\mathbb{R}^{3}$ :

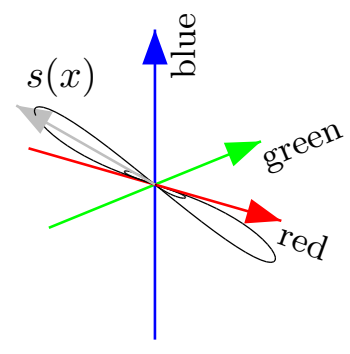

C: Embedding of $\mathbf{B}$ in the RGB cube:

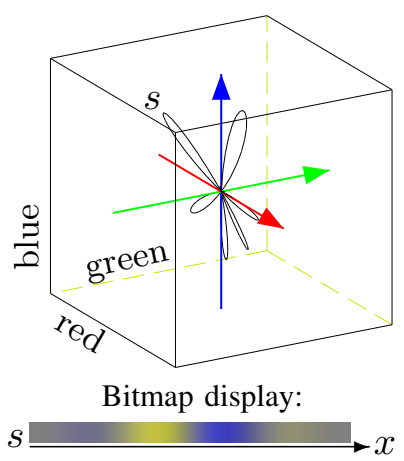

D: Color axis $\vec{u}$ on the unit sphere:

Fig. 5. Study of a color discontinuity: 1D example. Column (A) illustrates a color discontinuity in the RGB cube along the direction $\vec{u}$. Such a discontinuity is conveyed by an oscillation in the color space along the same direction $\vec{u}$ as shown in (B). This time $s(x)$ can be negative due to the band-pass filtering. Displaying this data by bitmaps like color images needs embedding it in the RGB cube (C), which is used to display 2D color subband and Riesz transform on figure 4. The basic Scilab code imshow (round $(255 *(0.5+0.5 * \mathrm{~s} / \mathrm{max}(\mathrm{abs}(\mathrm{s})))))$ ) provides the bitmap display of column (C). Particular colors involved in the discontinuity determine a certain 'color direction' $\vec{u}$ of oscillation that we encode into two angles $\alpha$ and $\beta$ as shown in column (D).

TABLE II

CORRESPONDENCE BETWEEN $\varphi_{2}$ AND SIGNAL SHAPE.

\begin{tabular}{|c|c|c|}
\hline $\operatorname{sgn}(s) / \varphi_{2}$ & 0 & $\frac{\pi}{2}$ \\
\hline+1 & & \\
\hline-1 & & \\
\hline
\end{tabular}

In the vector case, we can analogously split $s$ into its Euclidean norm $\|s\|$ and its 'color axis' ${ }^{4} \vec{u}$ :

$$
\vec{u}=s /\|s\|=\left[\begin{array}{lll}
\cos \alpha & \sin \alpha \cos \beta & \sin \alpha \sin \beta
\end{array}\right]^{\top}
$$

Color axis - illustrated figure 5 (D) - generalizes the sign data of the grayscale case and directly conveys a colorimetric feature of the original image's discontinuity (A).

The color monogenic model that we propose is defined as follows:

$$
s=\underbrace{\sqrt{\|s\|^{2}+\mathcal{N}^{2}}}_{A} \cos (\underbrace{\arg \{\|s\|+\boldsymbol{j} \mathcal{N}\}}_{\varphi_{2}}) \underbrace{\vec{u}}_{\text {'axis' }},
$$

where $\vec{u}$ indicates a direction in the $3 \mathrm{D}$ color space and $\varphi_{2}$ is the usual 1D phase. Gradient norm $\mathcal{N}$ is obtained with eq. (35). Finally, amplitude and phase can be retrieved with the sole Euclidean norm of $s$. The color extension brings two new components $(\alpha, \beta)$ corresponding to the local color axis along which $s$ oscillates.

\footnotetext{
${ }^{4}$ This is an arbitrary choice of spherical coordinate decomposition of $\vec{u}$.
}

The new color monogenic signal is built like a 4-vector whose spherical coordinates are amplitude, phase and color axis:

$$
\begin{aligned}
& s_{M}^{\text {color }}=\left[\begin{array}{llll}
s^{\mathrm{R}} & s^{\mathrm{G}} & s^{\mathrm{B}} & \mathcal{N}
\end{array}\right]^{\mathrm{T}} \\
& \text { Amplitude: } \quad A=\sqrt{\|s\|^{2}+\mathcal{N}^{2}} \in[0 ;+\infty[ \\
& \text { 1D Phase: } \quad \varphi_{2}=\arg \{\|s\|+\boldsymbol{j} \mathcal{N}\} \in\left[0 ; \frac{\pi}{2}[\right. \\
& \text { Color axis: } \quad\left\{\begin{array}{l}
\alpha=\arg \left\{s^{\mathrm{R}}+\boldsymbol{j} \sqrt{\left(s^{\mathrm{G}}\right)^{2}+\left(s^{\mathrm{B}}\right)^{2}}\right\} \in[0 ; \pi[ \\
\beta=\arg \left\{s^{\mathrm{G}}+\boldsymbol{j} s^{\mathrm{B}}\right\} \in[-\pi ; \pi[
\end{array}\right.
\end{aligned}
$$

It should be noticed that this is one direct way to estimate the 4 parameters of the model of equation (40) from the 9 available measures $\left[\begin{array}{lllllllll}s^{\mathrm{R}} & s^{\mathrm{G}} & s^{\mathrm{B}} & s_{x}^{\mathrm{R}} & s_{x}^{\mathrm{G}} & s_{x}^{\mathrm{B}} & s_{y}^{\mathrm{R}} & s_{y}^{\mathrm{G}} & s_{y}^{\mathrm{B}}\end{array}\right]$. It is in the line of the classical estimation of amplitude and phase with the scalar tools. Nevertheless, in a future work, a more general projection process will be studied.

Note that our method is consistant with the grayscale case - for which $\vec{u}=\operatorname{sgn}(s) \frac{1}{\sqrt{3}}[1,1,1]-$ and can be trivially generalized to $n$-vectors and hyperspectral images.

There is a very recent alternative approach toward 2-vector 1D analytic signal in [53]. The definition is different from ours as it implies a complex amplitude envelope with a complex instantaneous phase and is based on the 1D quaternion Fourier transform. However this work is limited to $1 \mathrm{D}$ complex signals and is not extendable to higher dimensions due to its specific embedding in the quaternion framework.

\section{E. Interpreting color monogenic analysis}

This new monogenic signal specially defined for multi-valued images consists in using the color Riesz norm $\mathcal{N}$ as the phase-shifted 


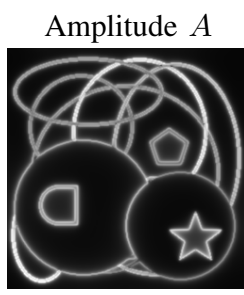

0

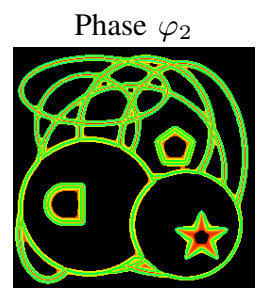

0
Color axis $\vec{u}$

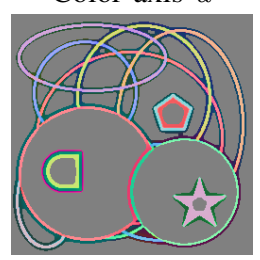

(Color pixels)
Fig. 6. Proposed color monogenic signal of image used in figure 4 . Here again, color axis and phase data are not displayed (black or gray) for coefficients with low amplitude.

version of $s$ given by $|\mathcal{R} s|$ in the scalar case. This magnitude is sufficient to have a complete reversible model through the 4 polar coordinates of eq. (41). The general phase is composed of 3 angles $(\varphi, \alpha, \beta)$, each one having a direct physical meaning in terms of amplitude, phase and color axis.

Note that orientation $\theta_{+}$from eq. (36) gives additional information that may be crucial for analysis purpose, but it is not part of the modulation model (see eq. 40). In fact the orientation analysis is intrinsic to the construction of the data $\varphi_{2}$ that only makes sense perpendicularly to the discontinuity's orientation. This ensures translation/rotation invariance and the well balanced response to all directions.

Let us observe the figure 6 illustrating our new color monogenic signal. Like previously, the analysis is done on a subband of the color image obtained with the same filter. We can see that amplitude is again consistent with geometrical structures (including isoluminant ones) and highlights oriented elements equally regardless of their orientation - due to Riesz transform isotropy. Its invariance to shift and rotation is due to the sharing of geometric information with $\varphi_{2}$ which forms a coherent coding. This also allows coding a line by a 'simple line' in amplitude instead of a 'double line' - as with gradient analysis ${ }^{5}$ like in figure 4 - thanks to the encoding of kind of discontinuity by $\varphi_{2}$ - already discussed section II-C. Orientation $\theta_{+}$is exactly this of figure 4. Angles $\alpha$ and $\beta$ - represented in the same graphic through $\vec{u}$ - carry some information of local color direction. They usually vary slowly up to sign changes, except around complex color structures involving two local color axes e.g. the green line separating the yellow region from the red disk. Note that our color generalization keeps all the desirable properties of the grayscale monogenic signal.

Based on this new extension of the monogenic signal, we can derive the corresponding color extension of the MWT presented section II-E.

\section{TENSOR BASED COLOR MONOGENIC WAVELET TRANSFORM}

We saw that the monogenic analysis must be done together with band-pass filtering that is selecting a particular scale for the analysis. In this section we generalize this tool to handle all scales in a global monogenic multiresolution analysis of color images.

\section{A. Definition}

The extension to the wavelet domain is direct since the above construction relies on a marginal Riesz transform (non marginality occurs when combining marginal outputs into meaningful data). So

\footnotetext{
${ }^{5}$ Derivative of an edge has a single response while derivative of a line has a double response. In practice, this can be efficiently handled with postprocessing but the signal approach unifies both kinds of discontinuities.
}

we can again directly use polyharmonic spline wavelets of Unser et al. (see section II-E) but with a different combination of Cartesian coefficients. This time components will be combined to carry out color AM/FM analysis.

We have first to compute the 6 following subband decompositions:

$$
\begin{aligned}
c_{i, \boldsymbol{k}}^{\mathrm{C}}= & \left\langle s^{\mathrm{C}}, \psi_{i, \boldsymbol{k}}\right\rangle=\left(\psi_{i} * s^{\mathrm{C}}\right)\left(2^{-(i+1)} \boldsymbol{k}\right) \\
d_{i, \boldsymbol{k}}^{\mathrm{C}}= & \left\langle s^{\mathrm{C}}, \psi_{i, \boldsymbol{k}}^{\mathcal{R}}\right\rangle=\left\{\mathcal{R}\left(\psi_{i} * s^{\mathrm{C}}\right)\right\}\left(2^{-(i+1)} \boldsymbol{k}\right) \\
& \text { with } \quad \mathrm{c} \in\{\mathrm{R}, \mathrm{G}, \mathrm{B}\}
\end{aligned}
$$

then to process Riesz norm:

$$
\mathcal{N}_{i, \boldsymbol{k}}=\sqrt{\left|d_{i, \boldsymbol{k}}^{\mathrm{R}}\right|^{2}+\left|d_{i, \boldsymbol{k}}^{\mathrm{G}}\right|^{2}+\left|d_{i, \boldsymbol{k}}^{\mathrm{B}}\right|^{2}}
$$

The proposed monogenic signal is for each scale $i$ :

$$
c_{M}(\boldsymbol{k})=\left[c_{i, \boldsymbol{k}}^{\mathrm{R}}, c_{i, \boldsymbol{k}}^{\mathrm{G}}, c_{i, \boldsymbol{k}}^{\mathrm{B}}, \mathcal{N}_{i, \boldsymbol{k}}\right]
$$

Amplitude, phase and color axis can be retrieved with eq. (41), and orientation with eq. (36). Computation load for the 6 decompositions is not serious since each one has a linear complexity. The figure 7 illustrates the multiscale color monogenic features obtained from our color monogenic wavelet transform.

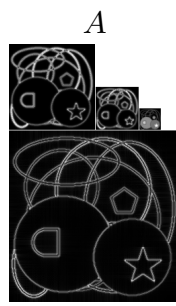

$0 \quad \max$
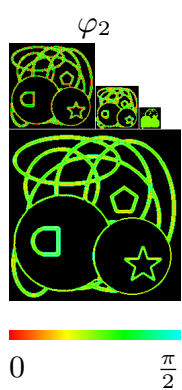

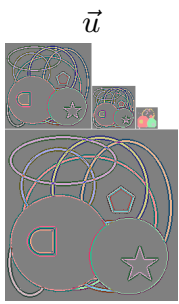

(Color axis)
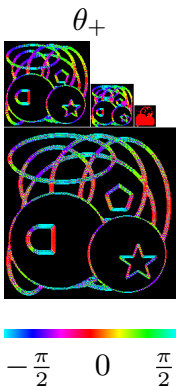

Fig. 7. The color monogenic wavelet transform $(\gamma=3)$.

\section{B. Continuous VS Discrete}

Before going into experimental use of this new representation, let us give some practical remarks.

Analytic/monogenic analysis must be thought in a multiscale scheme, this is why we focus on defining a monogenic wavelet transform. As already discussed section II-D, we could have chosen scalespace or PDE's that give a handy continuous multiscale tool. But we consider that signal processing tools are generally most relevant through such discrete schemes as filterbanks since they involve fast algorithms and provide compact data with each coefficient carrying much information, which is necessary for compression and real-time analysis.

The issue is that the monogenic analysis is basically defined in a continuous framework. Constraints related to filterbank design perfect reconstruction, small redundancy - conflict with desirable properties of isotropy and rotation invariance. The choice that is made by Unser et al. [5] is to provide the 'minimally-redundant wavelet counterpart of Felsberg's monogenic signal'. See also the Marr-like pyramid using the same algorithm in [54]. Our color monogenic wavelet transform is by extension in the same spirit.

Since filters cannot be exactly isotropic, the analysis is expected to mildly favor some directions. In addition, the subbands are highly subsampled (yet not 'critically' since number of coefficients is higher than number of pixels), implying that the phase data is varying fast with respect to sampling. Those two points slightly affect invariance properties of coefficients: this is the price to pay for 
having the reasonable redundancy of $\approx 1.8^{6}$. Fortunately, the implicit numerical implementation of the Riesz transform does not suffer from subsampling that appears outside the Riesz operator - see eqs. (42) and (43).

Now let us study how the color monogenic wavelet transform can be used. We will focus on sparsity and invariant analysis.

\section{Use as a sparse representation}

According to S. Mallat [55], sparse representations in redundant dictionaries can improve pattern recognition, compression, and noise reduction, but also the resolution of new inverse problems. This includes superresolution, source separation, and compressive sensing. As a redundant decomposition driven by the physical meaning of coefficients in terms of color and geometry, our multiscale color monogenic representation is a good candidate for sparsity. We expect that few coefficients will carry rich and meaningful visual information, so that we can select and quantize them simply without introducing serious artifacts.

We propose here to test the sparsity of this representation through simple selection and quantization of coefficients, together with some evaluation of the related distortion. This is also aimed at providing some insight into the interpretation of the wavelet domain data.

1) Unusual data: Quantization is usually optimized by statistical modeling - generalized Gaussian laws are often used for classical wavelets. In our case, usual tools hardly apply since our coefficients are in polar coordinate, basically a Euclidean norm and three angles. For example, an input Gaussian noise induces the amplitude to follow a Rayleigh distribution, as plotted figure 8. Usual wavelet

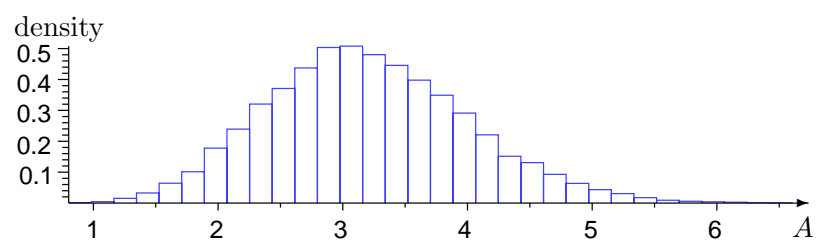

Fig. 8. Rayleigh distribution of amplitude of any subband for a Gaussian noise in input $(\gamma=3)$.

coefficients - in Cartesian coordinate - would follow a handier Gaussian distribution. This means that thresholding based denoising for example is not obviously transposed in our case by thresholding of amplitude. This also means that classical quantization schemes - optimized for classical wavelets - no longer apply. The issue is similar for the phase that is a circular data. Literature is quite poor about quantization of such data. The use of Von Mises distribution for example may help to statistically model the coefficients, but this is out of the scope of this paper.

2) Redundancy: Redundant representations may be thought of as computationally more expensive, however they often exhibit better sparsity. This is why undecimated wavelets for example have been successful in denoising and analysis [56]-[58]. Low-rate compression can also be better matched to the human visual system through redundant representations such as matching pursuit based dictionaries [59] or complex wavelets [60]. In a previous work [61] we highlighted that quaternionic analytic wavelets are more appropriate than classical

\footnotetext{
${ }^{6}$ Note that we speak about the redundancy of the wavelet domain data that is necessary to reconstruct the image, which is different from the redundancy of the 9D intermediate data composed of subbands and their Riesz transforms. Four pyramids of redundancy $4: 3$ to encode three color channels give $4 \times$ $\frac{4}{3} / 3=\frac{16}{9} \approx 1.8$. If we also count the orientation data - which is counted in Unser's proposition although not in the model - it raises to $\approx 2.2$. Anyway, since we have only one Riesz analysis for the three channels, redundancy is less than in the grayscale case.
}

biorthogonal wavelets in a quantization scheme because they introduce smoother artifacts. In particular, the quaternionic phase turns out to be robust against hard quantization. We expect the color monogenic transform to give analogous results for color images thanks to its quality of representation. We particularly assume that the information is significantly compressed.

3) Reconstruction: Wavelet domain processing requires the synthesis part of the filterbank. However, reconstruction from analytic/monogenic wavelet coefficients has not been dealt in the literature to our knowledge. The issue rises from the fact that there are several ways for the signal to be fully reconstructed, which also occurs with any redundant representation. In addition, the monogenic framework itself has this feature $\theta$ that is unused in the model $s=A \cos \varphi$ although it is part of the analysis. In our case, we choose to follow the 'modulation model' of eq. (3). This implies that the 'Riesz-Laplace' decomposition is not directly considered under analysis, but rather as intermediate data used to process an enriched description of 'primary' subbands composed of the 4 monogenic features $\left(A, \varphi_{2}, \alpha, \beta\right)$ at several scales. The monogenic wavelet domain processing consists in selecting and quantizing these 4 features - which are physically interpretable - rather than the raw wavelet coefficients $c^{\mathrm{C}}$ and $d^{\mathrm{C}}$. The corresponding modification of the 'primary' decomposition is obtained at a scale $i$ from:

$$
\left[\begin{array}{lll}
c^{\mathrm{R}} & c^{\mathrm{G}} & c^{\mathrm{B}}
\end{array}\right]=A \cos \varphi_{2}\left[\begin{array}{lll}
\cos \alpha & \sin \alpha \cos \beta & \sin \alpha \sin \beta
\end{array}\right]
$$

And the modified image comes from the synthesis part of the 'primary' filterbank applied on $\left(c^{\mathrm{R}}, c^{\mathrm{G}}, c^{\mathrm{B}}\right)$. The advantage of this method is that the 4 monogenic features are influent on the reconstructed image.

4) Experiments: In order to test the sparsity of our transform, we propose to observe partial reconstructions processed from monogenic wavelet domain uniform quantization. Note that an underlying selection of coefficients is done, since all values of $A$ in the first interval will be set to zero. Then eq. (46) implies that $A=0 \Rightarrow c^{R}=c^{G}=$ $c^{\mathrm{B}}=0$ - which means that all the corresponding phase values are also discarded. Depending on the number of bits used to quantize $A$, a certain thresholding will be done so that a limited number of (vector) coefficients will be retained. Together with this selection, each feature will be independently quantized to get an idea of the number of bits needed to properly code wavelet coefficients. This is also a way to better understand the kind of visual content that is coded by the various data.

We use 4 fixed numbers of bits $\left(B_{A}, B_{\varphi_{2}}, B_{\alpha}, B_{\beta}\right)$ for the whole transform, each one related to a feature. Decomposition level is 4 so that the scales are $i \in\{-1,-2,-3,-4\}$. The low frequency subband is not processed because it is not concerned by the monogenic analysis, and involves a negligible amount of data. We show on figure 9 (first column) that a uniform quantization on 6 bits (resp. 2, 3,4 ) for $A$ (resp. $\varphi_{2}, \alpha$ and $\beta$ ) induces almost no visible artifact in reconstructed image. This choice corresponds to a quantization step of $\pi / 8$ for the phase and color axis angles, which may be thought quite coarse. Total number of bits needed to code the image is then $16 / 9 \times(6+2+3+4) \approx 27$ times the number of pixels. This amount of data is comparable to the original 24 bit coding of raw color image.

Let us see the effect of applying a harder quantization on each component. By degrading amplitude $A$ (second column) we observe the classical pseudo-Gibbs effect introducing oscillations at borders of image circles. Textured areas are smoothed, like the lower left corner of mandrill or barbara's clothes. This confirms that $A$ is analogous to usual wavelet coefficients, which produce similar degradations. However, in our non-marginal scheme, color is not affected thanks to the separate coding of color axis. 


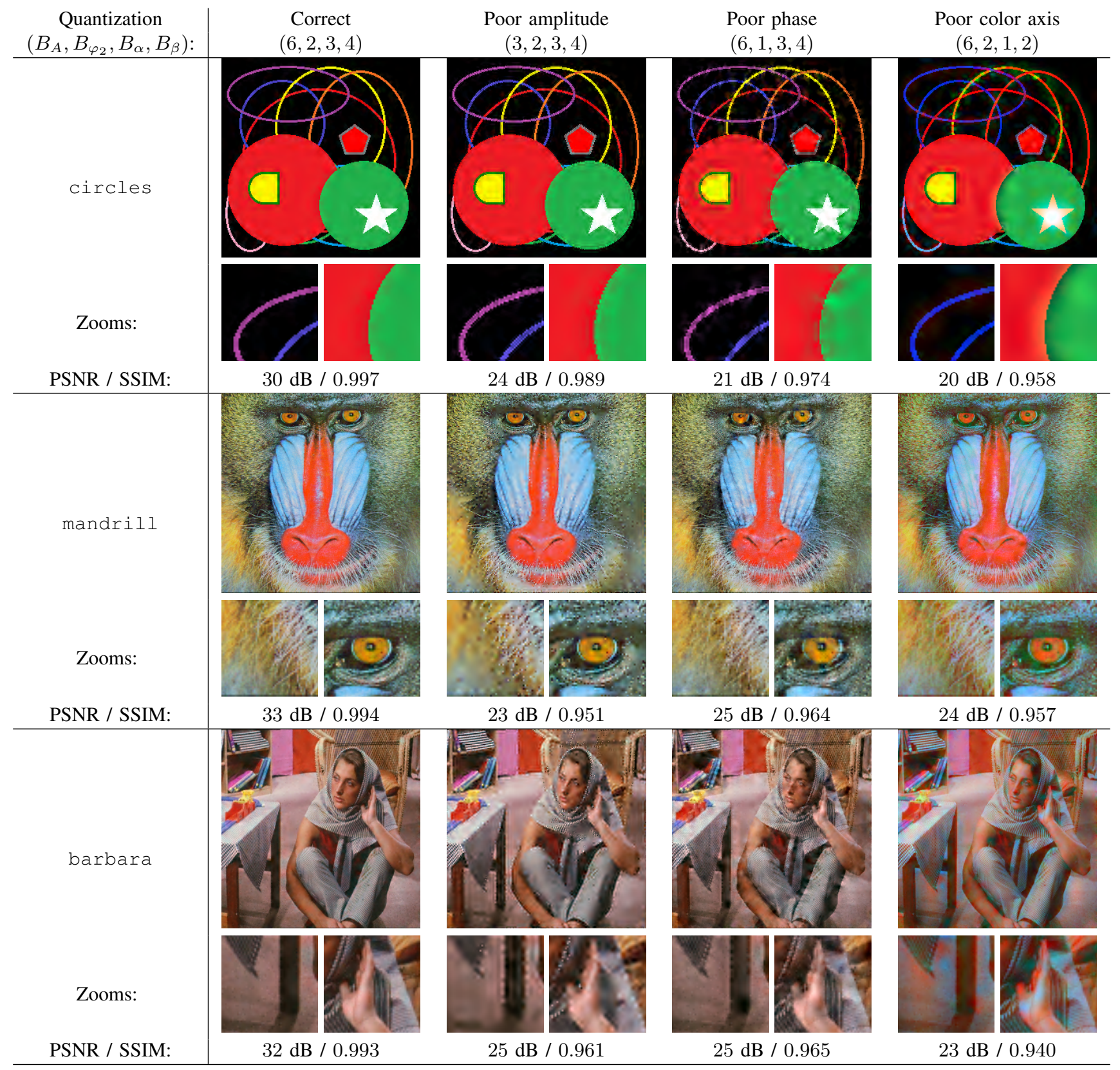

Fig. 9. Basic uniform quantization of $\left(A, \varphi_{2}, \alpha, \beta\right)$ on $\left(B_{A}, B_{\varphi_{2}}, B_{\alpha}, B_{\beta}\right)$ bits.

Hard quantization of the phase $\varphi_{2}$ produces visually annoying 'wet paper' effect. Phase error is particularly serious around discontinuities. Discontinuities are modeled like a sum of local oscillations at different frequencies. In the case of 'significant' discontinuity, those oscillations are expected to be locally in phase - this is the phase congruency that must exist at the maxima of amplitudes [9]. Quantization can badly increase small differences of phase between scales by rounding off on different neighboring centroids. This results in local bad alignments of different scales showing kinds of transparent oscillations around edges and lines. Note that phase error in a coarse scale coefficient implies a phase-shift on a large neighboring due to the coarse wavelet large support. See large oscillations at borders of circles or on table stand of barbara. In contrast, fine scale phase-shift artifacts are less significant - see that textures of mandrill are quite well preserved. Actually the phase degradation is mainly in low-frequencies, which explains this 'wet paper' effect that we already observed in [61] with quaternionic wavelets. Again, despite this strong degradation of contours, color is strikingly consistant like the green-red border between the big disks of circles.

Finally, by quantizing color axis only on $1+2=3$ bits we naturally introduce false colors. See for example mandrill's eyes, barbara's hand, or just any line of circles which color has totally changed. This confirms that $\alpha$ and $\beta$ encode some true colorimetric information being independent from $A$ and $\varphi_{2}$. Finer manipulation of the color content should be investigated through a deeper study of the color oscillation concept illustrated figure 5 .

This preliminary experiment shows that our transform is stable with quantization and can efficiently encode visually controlled color geometric elements. Discarding low-amplitude coefficients vectorwise does not introduce serious artifacts, due to the sparsity of this representation. We can independently control contours' sharpness and quality of colors with a reasonable number of bits. The originality of the work is that color images are handled in a unified framework 
revealing the color geometric content through interpretable coefficients. Our future work includes statistical modeling of monogenic coefficients in polar coordinate in order to optimize quantization. It will also be interesting to study the strong spatial correlation of color axis that is supposed to vary slowly, as well as the interscale correlation in order to reduce the data. Coefficients of small amplitude encode uniform areas which need not any geometric analysis. Their phase angles are not very significant so they may be quantized harder. Human visual system constraints such as orientation dependent weighting for example can be easily injected since an orientation analysis is accessible in the transform.

Let us now consider the color monogenic wavelet transform from the image analysis point of view.

\section{Invariant analysis of color images}

Wavelet based image analysis is generally aimed at handling several resolutions and directions e.g. for texture recognition or keypoint extraction. We propose here to perform basic analysis of wavelet coefficients to highlight their rotation invariance and better understand the information they carry. The great advantage of our method is that color is fundamentally taken into account.

The monogenic model is well fitted to analyze contours, through amplitude's local maxima. At those points where amplitude $A$ reaches a maximum, geometric analysis given by the phase and orientation are meaningful. It is shown in [9] (in the grayscale case) that amplitude's local maxima are equivalent to points of phase congruency giving efficient contour detection. Note that in the case of phase analysis, 'maximum' is understood in one direction, which includes saddle points as well. In practice, we will retain coefficients which amplitude is superior to at least 6 of their 8 neighbors. This tends to retain continuous thin contours, in contrast with usual keypoint detection that uses strict maxima in order to extract isolated corners and junctions.

To improve the invariance, we use the undecimated version of the transform as usually done e.g. for keypoint detection. Note that although the classical undecimated wavelet transform has been mostly used for denoising, it is also interesting for analysis, see [58], [62], [63]. This allows a better localization of features. Figure 10 shows analyses of a simple image rotated at three different angles. This illustration aims at confirming rotation invariance of the color monogenic analysis. The test image contains typical 'edges' and a 'line' that are elementary cases for the model.

1) Amplitude: We can see that the rotation does not change amplitude's shape, which experimentally confirms rotation invariance. Coefficients 'turn well' without any oscillation or artifact, thanks to the amplitude/phase representation. Like with the 1D Dual-Tree [34] or the 2D quaternionic wavelets [4], the amplitude gives a consistant representation of discontinuities analogous to some local energy. This provides a fine multiscale contour detection that handles well different kinds of discontinuities like lines and edges. A direct application of the amplitude $A$ could be a fast and efficient contour detection. This is the first phase based contour detection that can be done with multivalued images through a true counterpart of grayscale monogenic analysis and phase congruency.

For visual convenience, phase, color axis and orientation are displayed only at positions of amplitude's maxima.

2) Phase: Given the kind of discontinuity analysis detailed in table II, phase $\varphi_{2}$ is expected to have a value near 0 ('red') on the red line and around $\frac{\pi}{2}$ ('cyan') at the edges of the blue rectangle. At the coarsest scale $\varphi_{2}$ is consistant with our perception: 'red' on the line and 'cyan' on the edges. Corners are smoothed by the subband analysis so they are conveyed by curved edges, which are not handled in the monogenic framework. Invariance is perfect at the coarse scale, and very good at finer scales. For finer scales, the line tends to be considered as a thin rectangle. This is normal since the line's width is larger than one pixel so it can be viewed like either a line or a rectangle depending on the scale. At the finest scale, the analysis gives some rectangle shaped energy around the area of the line which is obviously conveyed by 'edges' $\left(\varphi_{2} \approx \frac{\pi}{2}\right)$. Actually, we rather observe 'green' phases $\left(\varphi_{2} \approx \frac{\pi}{4}\right)$ than 'blue'. The reason is low sampling density. Geometric analysis at the finest scale means studying 'one pixel wide' objects. Most of the times, objects of an image are not centered exactly on the grid. This issue is transposed in the monogenic analysis that has a sub-pixel accuracy so amplitude's maxima are also mostly between neighbor coefficients. The problem in this case is that $\varphi_{2}$ varies too fast with respect to the size of the object under analysis. We actually observe intermediate values of $\varphi_{2}$ - mostly 'green' phases - at finest scales, except at some 'cyan' points for which the edge fits well to the grid. Despite the sampling issue, phase appears clearly invariant to rotation.

3) Color axis: Amplitude and phase analysis (as well as orientation) have the advantage of being strictly analogous to their grayscale counterpart while being processed from a multi-valued image through a true vector geometric scheme. Remaining color information is independent to amplitude and phase and is encoded by the color axis. Color axis $\vec{u}$ is tricky to observe since it must be locally constant 'up to a sign change'. In the case of edges that are locally sin-shaped, a sign change always occurs at the central point. So we observe two values $\vec{u}_{1}$ and $\vec{u}_{2}=-\vec{u}_{1}$ coding the same axis in the color space. See also figure 5. This does not occur for lines that are cosshaped. In figure 10 , see that $\vec{u}$ is changing between 'orange' $\left(\vec{u}_{1}\right)$ and 'blue' $\left(\vec{u}_{2}=-\vec{u}_{1}\right)$ at edges, while really constant on the line. We actually have a representation issue about this data suggesting the use of 'modulo $\pi$ ' operators with $\alpha$ and $\beta$. Up to this sign change $\vec{u}$ is clearly invariant to rotation too.

This ensures that color information of image content is properly encoded through our monogenic analysis.

4) Orientation: Orientation analysis is expected to be invariant up to a constant offset $\theta_{0}$ equal to minus the angle of image's rotation. In the illustration we compensated this offset to make differences more visible. The finest scale suffers from the same sampling issue as $\varphi_{2}$. Differences appear around some orientations, due to both the approximate isotropy of filters, and the fact that orientation is estimated pointwise. Note that invariance can be improved by an additional smoothing of the Riesz tensor with Gaussian kernels as proposed by Unser et al. in [5] in the grayscale case. This modifies $\mathcal{N}$ and $\theta$ and thus the whole monogenic analysis, so that it is no longer done pointwise but within a neighborhood. This results in coefficients being more consistant with our perception, although this is not directly part of the monogenic framework. We show figure 11 color monogenic wavelets of rotated test images without and with Gaussian smoothing. Smoothing allows better orientation estimation and more stable amplitude's maxima. An additional illustration with a real world image is given on figure 12 .

5) Discussion: Finally, approximations related to the choice of a filterbank approach are acceptable, since we get experimental rotation invariance and correct physical interpretation of the yielded data. This monogenic wavelet representation of color images is consistent with the grayscale definition in terms of signal processing interpretation (amplitude, phase and orientation) as well as it takes advantage of a well defined differential model for handling vector signals.

Since existing wavelet transforms are fundamentally of a different nature (known analytic wavelets are grayscale only, known color wavelets are not analytic), and existing wavelet based applications do not apply to monogenic coefficients, it is quite impossible to 


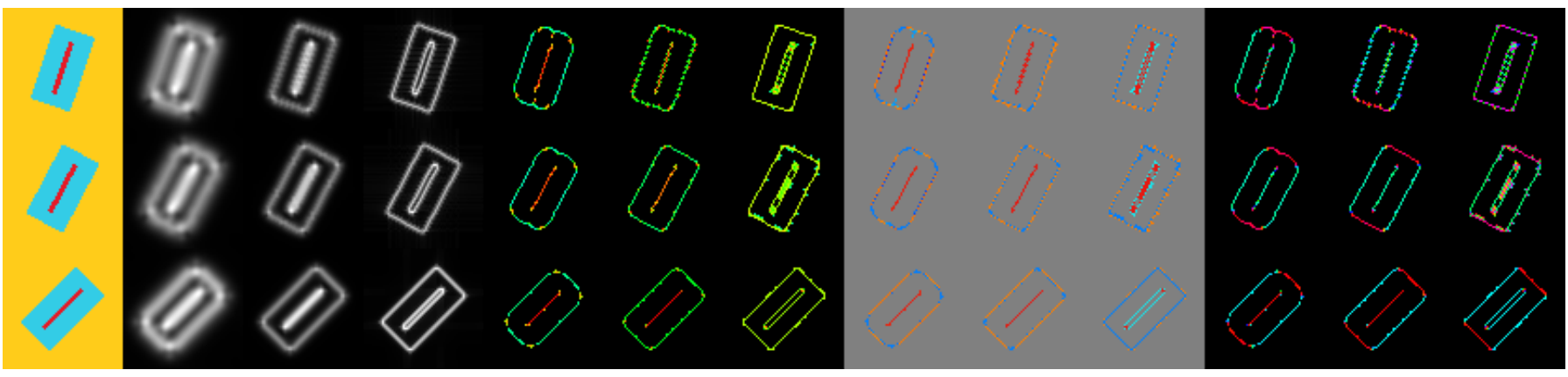

Fig. 10. Undecimated color monogenic wavelet decomposition $(\gamma=3)$ for several rotated versions of a simple test image (one per row). From left to right: Test image, amplitudes for 3 scales from coarsest to finest, phase $\varphi_{2}$, color axis and orientation for each scale. Phase/Axis/Orientation values are displayed only at positions of amplitude's local maxima. Orientation has been offset by a constant angle to compensate image rotation. Legend is the same as figure 7.

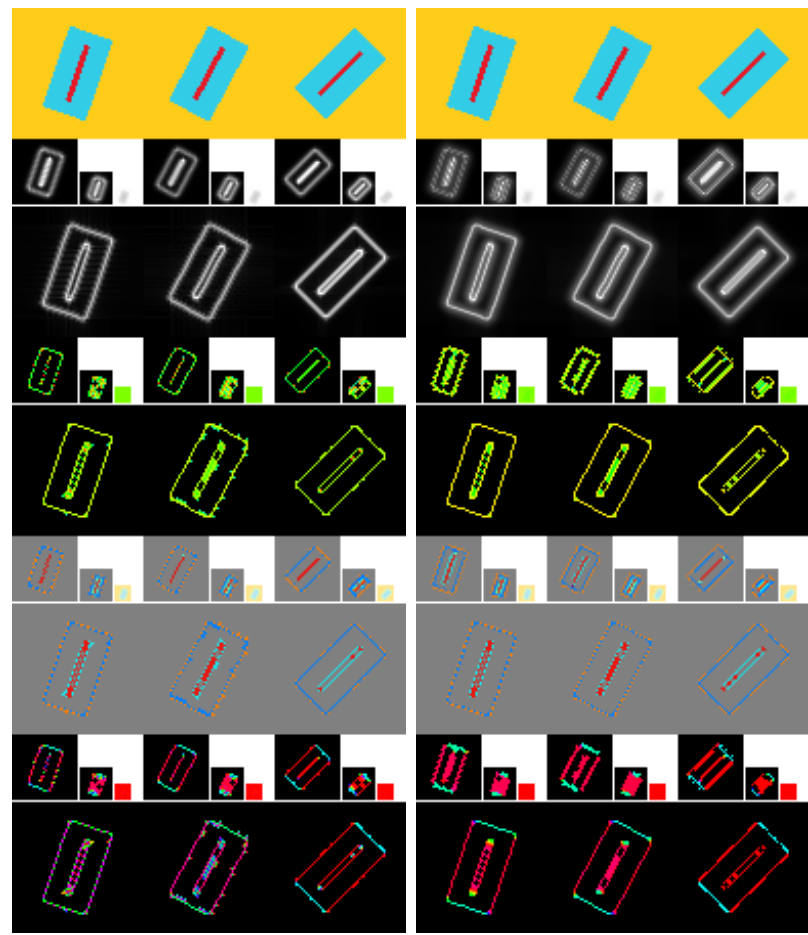

Fig. 11. Rotation variance of color monogenic wavelet transform without (left) and with (right) Gaussian smoothing of the Riesz tensor. $\gamma=3$ and the Gaussian kernel is $\sigma=1.5$.

compare our transform with the literature without carrying out a whole competitive application. Our present work is the basis of a new keypoint detector for color images through a fundamental geometric analysis of the color content with new invariance properties and a multiscale flavor. It paves the way to the definition of new invariant descriptors based on local amplitude, phase, orientation and color axis to fully characterize color keypoints. This would constitute a competitive color alternative to the famous SIFT detector [64], by introducing physical interpretation through 'color phase' and 'color frequency' notions. This signal flavor will allow getting closer to the human visual system. Our monogenic coefficients should also provide good texture classification through statistical features as it is already the case for grayscale quaternionic wavelets [61].

\section{CONCLUSION}

Concepts of multiscale analytic/monogenic signal and local phase have been gaining much interest since they tend to provide physically interpretable representations while being linked to flexible unifying algebraic frameworks. This paper presents an innovative construction of non-marginal color monogenic wavelets. Such a geometric representation allows the color content of images to be coherently encoded by meaningful data. This is the first time that color is considered in such a wavelet transform so that no false color is introduced and all contours are modeled - even isoluminant ones.

The construction is based on a theoretical link between the Riesz transform, building block of the monogenic framework - and the gradient, basis of the structure tensor. Thanks to vector differential geometry, we build a color phase concept tied to a non-marginal color extension of the grayscale monogenic signal. The efficient tensorbased geometric analysis - which is responsible for the success of PDE's based color regularization by intrinsically considering color distances - is joined to the physical amplitude/phase modeling, carrying out a unified representation of color images through amplitude, phase, orientation and color axis data. These features are invariant or covariant to shifts and rotation.

The wavelet transform is constructed from the grayscale monogenic wavelets of Unser et al [5] by extending it according to our nonmarginal color definition. The resulting transform is non-separable and slightly redundant $(16: 9 \approx 1.8)$ so that isotropy and rotation invariance are nearly achieved.

The amplitude data indicates the local energy (local in both space and frequency) similar to the modulus of usual wavelet coefficients, and having the same kind of sparsity (A few high coefficients contain most of the total energy). It performs an efficient multiscale contour detection unifying color lines and edges without any preferred spatial direction and handling all color contours. It is responsible for the quality of textured areas and the global structure of the image.

Maxima of amplitude reveal multiscale keypoints for which values of phase, orientation and color axis give geometric and colorimetric information:

- The phase data gives the local contour type, already studied through the concept of phase congruency yielding a continuous model between 'lines' and 'edges'. It controls the coherency of contours.

- The color axis carries the color information related to discontinuities, allowing controlling the quality of color independently from the geometric structure of the image.

- Orientation is equivalent to a multiscale gradient direction that is embedded in the monogenic framework. It can be used for example to take into account the human visual system, but remains an additional data with no impact on the reconstruction.

The whole data is unified in our color monogenic framework so as to carry out consistant and complementary low-level features.

This elegant distribution of the information allows a sparse representation, where most coefficients have low amplitude and thus insignificant phase and color axis. We illustrate this sparsity through 


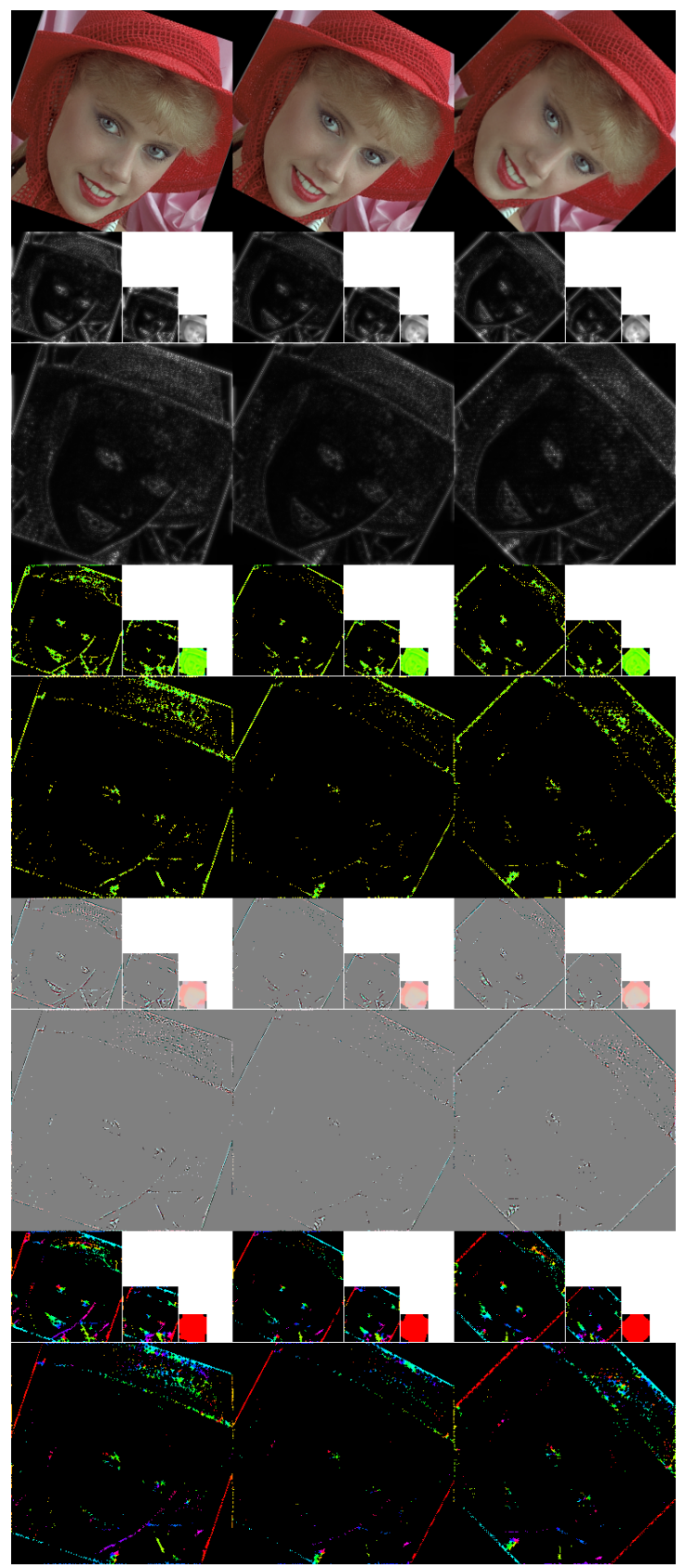

Fig. 12. Rotation variance of color MWT for a real image $(\gamma=3)$.

quantization experiments revealing the visual information carried by the different coefficients. Geometric multiscale analysis of color images is also investigated through invariant keypoint detection. While the famous SIFT algorithm is widely used for its invariance properties, we introduce the basis of a new method where color is naturally handled and physical interpretation of the data is possible thanks to our signal processing approach.

Our future work will include defining higher level local descriptors to fully characterize the keypoints, as well as integrating human visual system tools for interest point detection. The fundamental handling of color information that we propose is very useful for such applications. Theoretical prospects include consideration of other color spaces than RGB in the definition of color phase, and a more general reflexion about the estimation of the monogenic features as well as the reconstruction method. The numerical aspect of the monogenic analysis also has to be further studied, and possibly non redundant schemes may be investigated. In addition, a unifying algebraic embedding analogous to complex Fourier analysis in the 1D scalar case could carry out an elegant signal processing framework adapted to color images. It would also be interesting to look at the other monogenic wavelets that have been proposed in the grayscale case. The construction can easily be extended to the general multichannel and multispectral case by considering general vector structure tensors. Direct applications are sparse representation and classification of multispectral images.

\section{ACKNOWLEDGMENT}

We would like to thank the anonymous reviewers whose numerous remarks greatly contributed to this paper. We also thank Dimitri Van De Ville and Michael Unser from the EPFL for kindly having given us their source code of the polyharmonic spline wavelet pyramid. This work is part of the French ANR project VERSO-CAIMAN. We also thank the French Poitou-Charentes region for participating.

\section{REFERENCES}

[1] L. Jacques, L. Duval, C. Chaux, and G. Peyré, "A panorama on multiscale geometric representations, intertwiningspatial, directional and frequency selectivity," Sig. Proc., vol. 91, no. 12, pp. 2699 - 2730, 2011.

[2] T. Bülow, "Hypercomplex spectral signal representation for the processing and analysis of images," Thesis, August 1999.

[3] M. Felsberg and G. Sommer, "The monogenic signal," IEEE Trans. Signal Process., vol. 49, no. 12, pp. 3136-3144, 2001.

[4] W. L. Chan, H. H. Choi, and R. G. Baraniuk, "Coherent multiscale image processing using dual-tree quaternion wavelets," IEEE Trans. Image Process., vol. 17, no. 7, pp. 1069-1082, 2008.

[5] M. Unser, D. Sage, and D. Van De Ville, "Multiresolution monogenic signal analysis using the riesz-laplace wavelet transform," IEEE Trans. Image Process., vol. 18, no. 11, pp. 2402-2418, 2009.

[6] J. Ville, "Théorie et applications de la notion de signal analytique," Cables et transmission, vol. 2A, no. 1, pp. 61-74, 1948.

[7] D. Gabor, "Theory of communication," J. Inst. Elec. Eng., vol. 93, no. 3, pp. 429-457, 1946.

[8] A. Oppenheim and J. Lim, "The importance of phase in signals," Proc. IEEE, vol. 69, no. 5, pp. 529-541, 1981 .

[9] M. C. Morrone and R. A. Owens, "Feature detection from local energy," Pat. Rec. Lett., vol. 6, no. 5, pp. 303-313, 1987.

[10] P. Kovesi, "Image features from phase congruency," Videre: J. Comp. Vis. Research, vol. 1, no. 3, 1999.

[11] G. H. Granlund and H. Knutsson, Signal processing for computer vision. Kluwer, 1995.

[12] P. Kovesi, "Edges are not just steps," in Proc. Asian Conference on Computer Vision (ACCV'O2), Jan. 2002, pp. 822-827.

[13] J. P. Havlicek, D. S. Harding, and A. C. Bovik, "Multidimensional quasi-eigenfunction approximations and multicomponent am- $\mathrm{fm}$ models," IEEE Trans. Image Process., vol. 9, no. 2, pp. 227-242, 2000.

[14] K. Larkin, D. Bone, and M. Oldfield, "Natural demodulation of two dimensional fringe patterns: I. general background to the spiral phase quadrature transform," J. Opt. Soc. Am., vol. 18, pp. 1862-1870, 2001.

[15] E. W. Stein and G. Weiss, Introduction to Fourier Analysis on Euclidean Spaces. Princeton Univ. Press, NJ, 1971.

[16] S. C. Olhede and G. Metikas, "The monogenic wavelet transform," IEEE Trans. Signal Process., vol. 57, pp. 3426-3441, 2009.

[17] S. Held, M. Storath, P. Massopust, and B. Forster, "Steerable wavelet frames based on the riesz transform," IEEE Trans. Image Process., vol. 19, no. 3, pp. $653-667,2010$.

[18] A. C. Bovik, Handbook of Image and Video Processing. Academic Press, May 2000.

[19] G. Demarcq, L. Mascarilla, and P. Courtellemont, "The color monogenic signal: a new framework for color image processing" in Proc. IEEE Int'l Conf. on Image Processing, 2009.

[20] R. Soulard and P. Carré, "Color Monogenic Wavelets for Image Analysis" in Proc. IEEE Int'l Conf. on Image Processing, 2011.

[21] S. Di Zenzo, "A note on the gradient of a multi-image," Computer Vision, Graphics, and Image Processing, vol. 33, no. 1, pp. 116 - 125, 1986.

[22] D. Tschumperlé and R. Deriche, "Vector-valued image regularization with PDEs: A common framework for different applications," IEEE Trans. Pattern Anal. Mach. Intell., vol. 27, no. 4, pp. 506-517, 2005. 
[23] N. Sochen, R. Kimmel, and R. Malladi, "A general framework low level vision," IEEE Trans. Image Process., vol. 7, pp. 310-318, 1998.

[24] A. Benazza-Benyahia, J.-C. Pesquet, and M. Hamdi, "Vector-lifting schemes for lossless coding and progressive archival of multispectral images," IEEE Trans. Geosci. Remote Sens., vol. 40, no. 9, 2002.

[25] V. Labunets, A. Maidan, E. Labunets-Rundblad, and J. Astola, "Colour triplet-valued wavelets and splines," in Proc. Int'l Symp. Im. Sig. Proc. Analysis (ISPA'01), Pula, Croatia, Jun. 2001, pp. 535 - 540.

[26] M. Vetterli and G. Strang, "Time-varying filter banks and multiwavelets," in IEEE DSP Workshop, 1994, pp. 223-226.

[27] P. Carré and P. Denis, "Quaternionic Wavelet Transform for Colour Images," in Proc. SPIE, Wavelet Applications in Industrial Processing $I V$, vol. 6383, 2006.

[28] P. Denis, P. Carré, and C. Fernandez-Maloigne, "Spatial and spectral Quaternionic approaches for Colour Images," Computer Vision and Image Understanding, vol. 107, no. 1-2, pp. 74-87, 2007.

[29] T. A. Ell and S. J. Sangwine, "Hypercomplex fourier transforms of color images," IEEE Trans. Image Process., vol. 16, no. 1, pp. 22-35, 2007.

[30] S. L. Hahn, "Multidimensional complex signals with single-orthant spectra," Proc. IEEE, vol. 80, no. 8, pp. 1287-1300, 1992.

[31] C. T. Nguyen, J. D. Williams, J. P. Havlicek, and M. Özaydin, "FM processing with generalized amplitude and phase: Application to modulation domain geometric image transformations," in Proc. IEEE Int'l Conf. on Image Processing. Brussels (Belgium), Sep. 2011, pp. 81-84.

[32] O. Fleischmann, L. Wietzke, and G. Sommer, "Image analysis by conformal embedding," J. of Math. Im. and Vis., vol. 40, no. 3, pp. 305-325, 2011.

[33] M. Felsberg and G. Sommer, "The monogenic scale-space : A unifying approach to phase-based imageprocessing in scale-space," J. of Math. Im. and Vis., vol. 21, no. 1, pp. 5-26, 2004.

[34] I. Selesnick, R. Baraniuk, and N. Kingsbury, "The dual-tree complex wavelet transform - a coherent framework for multiscale signal and image processing," IEEE Sig. Proc. Mag., vol. 22, no. 6, 2005.

[35] E. Bayro-Corrochano and M. A. De La Torre Gomora, "Image processing using the quaternion wavelet transform," in Proc. CIARP, Puebla, Mexico, 2004, pp. 612-620.

[36] J. Zhou, Y. Xu, and X. Yang, "Quaternion wavelet phase based stereo matching for uncalibrated images," Pat. Rec. Lett., vol. 28, no. 12, 2007.

[37] Z. Yu, Y. Xu, and X. Yang, "Fire surveillance method based on quaternionic wavelet features," in MMM, 2010, pp. 477-488.

[38] R. Soulard and P. Carré, "Quaternionic wavelets for texture classification," Pat. Rec. Lett., vol. 32, no. 13, pp. 1669 - 1678, 2011.

[39] D. Van De Ville, T. Blu, and M. Unser, "Isotropic polyharmonic bsplines: Scaling functions and wavelets," IEEE Trans. Image Process., vol. 14 :(11), pp. 1798-1813, 2005

[40] R. Duits, M. Felsberg, L. Florack, and B. Platel, " $\alpha$-scale spaces on a bounded domain," in Proc. Scale-Space, LNCS 2695. Berlin, Heidelberg: Springer, 2003, pp. 494-510.

[41] M. Unser and D. Van De Ville, "The pairing of a wavelet basis with a mildly redundant analysis via subband regression," IEEE Trans. Image Process., vol. 17, no. 11, pp. 1-13, 2008.

[42] J. Li, H. Zhao, X. Zhou, and C. Shi, "Robust stereo image matching using a two-dimensional monogenic wavelet transform," Opt. Lett., vol. 34, no. 22, pp. 3514-3516, 2009.

[43] C. E. Moxey, S. J. Sangwine, and T. A. Ell, "Hypercomplex correlation techniques for vector images," IEEE Trans. Signal Process., vol. 51, no. 7, pp. 1941-1953, 2003.

[44] M. Felsberg and U. Köthe, "Get: The connection between monogenic scale-space and gaussian derivatives," in Proc. Scale-Space, LNCS 3459, Springer, 2005, pp. 192-203

[45] U. Köthe and M. Felsberg, "Riesz-transforms versus derivatives: On the relationship between the boundary tensor and the energy tensor," in Proc. Scale-Space, LNCS 3459, Springer, 2005, pp. 179-191.

[46] B. Jähne, Digital Image Processing 6th Edition. Berlin: Springer, 2005.

[47] J. Bigun, G. Granlund, and J. Wiklund, "Multidimensional orientation estimation with applications to texture analysis and optical flow," IEEE Trans. Pattern Anal. Mach. Intell., vol. 13, no. 8, pp. 775-790, 1991.

[48] U. Köthe, "Edge and junction detection with an improved structure tensor," in Proc. DAGM Symposium, LNCS 2781. Berlin, Heidelberg: Springer, 2003, pp. 25-32.

[49] M. Felsberg, "Low-level image processing with the structure multivector," Thesis, 2002.

[50] G. Sapiro and D. L. Ringach, "Anisotropic diffusion of multivalued images with applications to color filtering," IEEE Trans. Image Process., vol. 5, no. 11, pp. 1582-1586, 1996.

[51] R. Deriche, "Using Canny's criteria to derive a recursively implemented optimal edge detector," Int'l J. of Comp. Vis., vol. 1, no. 2, 1987.
[52] M. Felsberg and G. Sommer, "Image features based on a new approach to $2 \mathrm{~d}$ rotation invariant quadrature filters," in Proc. Europ. Conf. Comp. Vis. (ECCV'02), LNCS 2350, A. Heyden, G. Sparr, M. Nielsen, and P. Johansen, Eds., 2002, pp. 369-383.

[53] N. Le Bihan and S. Sangwine, "Quaternionic spectral analysis of non stationary improper complex signals." in Proc. Int'l Conf. Clifford Algebras and Appli. in Math. Physics (ICCA9), Jul. 2011.

[54] D. Van De Ville and M. Unser, "Complex wavelet bases, steerability, and the marr-like pyramid," IEEE Trans. Image Process., vol. 17, no. 11, pp. 2063-2080, 2008.

[55] S. Mallat, A Wavelet Tour of Signal Processing, Third Edition: The Sparse Way, 3rd ed. Academic Press, Dec. 2008.

[56] R. R. Coifman and D. L. Donoho, "Translation-invariant de-noising," Yale University and Stanford University, Tech. Rep., 1995.

[57] W. L. Hwang and S. Mallat, "Characterization of self-similar multifractals with wavelet maxima," Appl. Comp. Harm. Anal., vol. 1, no. 4, pp. 316-328, 1994.

[58] J.-L. Starck, J. M. Fadili, and F. Murtagh, "The undecimated wavelet decomposition and its reconstruction," IEEE Trans. Image Process., vol. 16, no. 2, pp. 297-309, 2007.

[59] R. M. Figueras i Ventura, P. Vandergheynst, and P. Frossard, "Low-rate and flexible image coding with redundant representations," IEEE Trans. Image Process., vol. 15, no. 3, pp. 726-739, 2006.

[60] J.-Y. Yang, J. Xu, F. Wu, Q. Dai, and Y. Wang, "Image coding using 2-d anisotropic dual-tree discrete wavelet transform," in Proc. IEEE Int'l Conf. on Image Processing, 2007, pp. 165-168.

[61] R. Soulard and P. Carré, "Quaternionic wavelets for image coding," in Proc. Europ. Sig. Proc. Conf. (EUSIPCO'10), Aalborg, Denmark, Aug. 2010, pp. 125-129.

[62] M. Khansari, H. R. Rabiee, M. Asadi, and M. Ghanbari, "Object tracking in crowded video scenes based on the undecimated wavelet features and texture analysis," EURASIP J. Adv. Sig. Proc., 2008.

[63] G. Mirajkar and B. Barbadekar, "Automatic segmentation of brain tumors from MR images using undecimated wavelet transform and gabor wavelets," in Proc. IEEE Elec. Circ. Syst., 2010, pp. 702-705.

[64] D. G. Lowe, "Distinctive image features from scale-invariant keypoints," Int'l J. of Comp. Vis., vol. 60, no. 2, pp. 91-110, 2004.

Raphaël Soulard received the PhD degree in 2012 from the University of Poitiers, France. He is currently a postdoctoral research fellow at the XLIMSIC laboratory, France. His research interests include wavelets and filterbanks, analytic/monogenic representation methods, extension of signal and image processing tools for multidimensional/multivalued signals like color images. He received the national prize "Blanc-Lapierre" granted by the SEE (Society of the electricity, the electronics, and the information technologies) for his master's thesis in 2009 .

Philippe Carré received the Engineer degree in Computer Engineering from Compiegne University of Technology, France, in 1995, and the Ph.D. degree in Signal Processing and Computer Science in University of Poitiers, France, in 2000 for his work on the definition of discrete atomic Time-Frequency representations. He is presently Professor at the XLIM-SIC laboratory, France. His interests include signal and image processing, wavelets, Time-Frequency and multiscale decompositions theory and their applications to the fields of denoising, segmentation and watermarking.

Christine Fernandez-Maloigne is currently the director of the XLIM-SIC laboratory, France, as well as co-manager of a regional research federation (MIRES), which gathers 560 researchers in region Poitou-Charentes, in France, in the area of mathematics, image processing, computer graphic, computer science and communication systems. Christine Fernandez-Maloigne has authored over 200 articles in the areas of colour imaging. Christine Fernandez-Maloigne is member of the National Committee of CNRS since 2012. She is also French representative of the CIE Division 8 where she manages a Technical committee about image and video compression quality assessment (TC8-12). 